\title{
From Diofantian Equations to Matricial Equations (I) - Equations and Pythagorean Matrices
}

\author{
Teodor Dumitru Vălcan
}

\begin{abstract}
We propose, starting with this work, presenting a way to achieve a systemic vision of a certain mathematical notional content, vision to motivate and mobilize the work of those who teach in class, thus facilitating teaching and assimilation of notions, concepts, scientific theories approached by educational disciplines that present phenomena and processes in nature. We present here a systemic approach to solving a Diophantine equation, namely a Pythagorean equation in the set of natural numbers, then in $\mathbf{Z}$, to then "submerged" such an equation in a ring of matrices and try to find as many matrices solutions as possible. Thus, first we will present a didactic solution to the Pythagorean equation, after which we will determine all the matrix solutions of such an equation in the $M$ ring. These matrices will be called pitagorean matrices. We will limit ourselves only to the second-order matrices, because for larger matrices the calculations are more and more complicated. But we will also present matrix solutions of at least 3.
\end{abstract}

Keywords: equation, system, solution, matrix, ring.

\section{Introduction}

Mathematics, abstract and open science, is the same throughout the world. It does not support interpretations other than within it, and they are related to the systemic structures that are being adopted to decide the curriculum (here in the meaning of the Mathematics curriculum, but also in terms of the objectives of mathematical education, the chosen methodology and the evaluation criteria).

Therefore, the design of the training activity includes, in the analysis of educational and instructive resources, the systemic processing of the notional content, a didactic operation that reveals the teacher's ability to understand and structure the scientific information in order to make it more accessible to the pupils.

We have to start, however, from the premise that the current conditions of the educational process, no matter where it's going, is characterized by the following three fundamental features:

a) The "informational assault" of the new scientific discoveries in the field of school subjects having as object of study the nature and reconsider some points of view of established theories, outdated the emergence of new ideas, concepts or simple notions, theories with an integrating character and including the first ones. All these require permanent restructurings and re-evaluations of the contents of the respective school disciplines, to adapt to the "new" by reformatting the programs and textbooks.

b) Achieving "content accessibility" taught to the educational disciplines. This is done taking into account the theories of learning, elaborated by psycho-pedagogical research. In this case it's recording progress and developments and by the intake of new disciplines, such as Cognitive Psychology.

c) The contribution of teachers. Those who work in education, directing the development of the didactic process, to be able to cope with the rapid developments outlined above, are being readied at different rhythms, using various sources of information, sometimes contradictory or outdated, remaining tributaries, many times, outdated mentalities about the nature, quantity and quality of scientific information offered in teacher-student communication, on the distribution of the roles and responsibilities of those who participate in this communication, as well as the aims pursued through education and education in general.

Here's why, we propose, starting with this paper, presenting a way to achieve a systemic vision of a certain mathematical notional content, vision to motivate and mobilize the work of those who teach in class, thus facilitating teaching and assimilation of notions, concepts, scientific theories approached by educational disciplines that present phenomena and processes in nature.We begin with a systemic approach to solving a Diophantine equation, more precisely of a Pythagorean equation in the set of natural numbers, then in $\mathbf{Z}$, to then "submerged" such an equation in a ring of matrices and try to find as many matrices solutions as possible.

\section{Solving a Pythagorean Equation}

In this paragraph we consider the Pythagorean equation:

$$
\mathrm{x}^{2}+\mathrm{y}^{2}=\mathrm{z}^{2} \text {, }
$$


which we will try to solve in a didactic style, so that this can be done with the students in the classroom.Studied in detail by Pitagora in relation to the rectangular triangles whose sides have the lengths of natural numbers, this equation has been known since the time of the old Babylonians.

For starters, three remarks are necessary here:

Remark 2.1: If the triplet $\left(x_{0}, y_{0}, z_{0}\right) \in \mathbf{N} \times \boldsymbol{N} \times \boldsymbol{N}$ satisfies equation (A), then any triplet of form $\left(k \cdot x_{0}, k \cdot y_{0}, k \cdot z_{0}\right)$, with $k \in \mathbf{Z}$, is a solution of this in $\mathbf{Z} \times \mathbf{Z} \times \mathbf{Z}$. Therefore, to solve equation (A), it is sufficient to determine solutions $(x, y, z) \in \mathbf{N} \times \mathbf{N} \times \mathbf{N}$ with the property that:

$$
(x, y, z)=1 \text {. }
$$

This is in fact equivalent to determining the solutions $(\mathrm{x}, \mathrm{y}, \mathrm{z})$ for which the numbers $\mathrm{x}, \mathrm{y}$ and $\mathrm{z}$ are two by two prime ones.

Here's why the following definition is required:

Definition 2.2: A solution $\left(x_{0}, y_{0}, z_{0}\right) \in \boldsymbol{N}^{*} \times \boldsymbol{N}^{*} \times \boldsymbol{N}^{*}$ of equation (A) such that the numbers $x_{0}, y_{0}$ and $z_{0}$ are two by two primes between them is called the primitive solution.

Remark 2.3: The triplets $(0, x, x)$ and $(x, 0, x)$ with $x$ natural number are solutions of equation $(A)$.

Therefore, further, we will only consider solutions with non-zero components.

Remark 2.4: Equation (A) is symmetric in $x$ and $y$; so if $(x, y, z) \in N^{*} \times N^{*} \times N^{*}$ is the solution of it, then $(y, x, z) \in N^{*} \times N^{*} \times N^{*}$ is also the solution of the equation (A).

We present below the form of a primitive solution of equation (A):

Theorem 2.5: Any primitive solution $\left(x_{0}, y_{0}, z_{0}\right)$ in nonzero natural numbers of equation $(A)$ is the form:

$$
\begin{array}{lll}
x=a^{2}-b^{2}, & y=2 \cdot a \cdot b \quad \text { and } \quad z=a^{2}+b^{2},
\end{array}
$$

With $a$ and $b$ natural numbers, relatively prime and $a>b$.

Proof: Therefore, according to Remark 2.1, the numbers $\mathrm{x}, \mathrm{y}$ and $\mathrm{z}$ can not all be even. We distinguish the following cases:

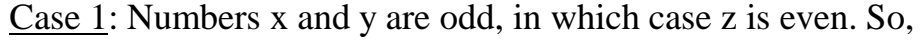

$$
\mathrm{x}=2 \cdot \mathrm{m}+1, \quad \mathrm{y}=2 \cdot \mathrm{n}+1 \quad \mathrm{z}=2 \cdot \mathrm{p},(2.2)
$$

with $\mathrm{m}, \mathrm{n}, \mathrm{p} \in \mathbf{N}^{*}$. Then, equality (A) becomes:

$4 \cdot m \cdot(m+1)+4 \cdot n \cdot(n+1)+2=4 \cdot p^{2}$,

what is impossible modulo 4 (the right member is only a multiple of 2 and the left member is a multiple of 4).

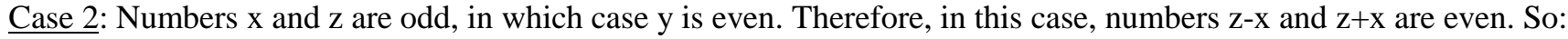
$\mathrm{z}+\mathrm{x}=2 \cdot \mathrm{m}$,
$\mathrm{y}=2 \cdot \mathrm{n}$
and
$\mathrm{z}-\mathrm{x}=2 \cdot \mathrm{p}$,

with $\mathrm{m}, \mathrm{n}, \mathrm{p} \in \mathbf{N}^{*}$. Since $\mathrm{z}$ and $\mathrm{x}$ are prime numbers between them, it follows that the numbers $\mathrm{m}$ and $\mathrm{p}$ are (also) prime between them, that is:

$(\mathrm{m}, \mathrm{p})=1$.

On the other hand, from equality (A), it follows that: $n^{2}=m \cdot p$.

Now, from equalities (2.5) and (2.6), it follows that there are numbers $a, b \in \mathbf{N}^{*}$, relatively prime, so that:

$$
\mathrm{m}=\mathrm{a}^{2}, \quad \mathrm{p}=\mathrm{b}^{2} \quad \text { and } \mathrm{n}=\mathrm{a} \cdot \mathrm{b}
$$

Finally, from equalities (2.4) and from equality (A), it follows equalities (*).

Otherwise: We write equation (A) thus:

$$
(z-y) \cdot(z+y)=x^{2}
$$

and how $\mathrm{y}$ and $\mathrm{z}$ are relatively prime numbers, it follows that:

$(\mathrm{z}-\mathrm{y}, \mathrm{z}+\mathrm{y})=1$.

From equalities $\left(\mathrm{A}^{\prime}\right)$ and (2.8) and from the uniqueness of decomposition in primitive factors of the number $\mathrm{x}^{2}$, it follows that there are odd numbers $\alpha, \beta \in \mathbf{N}$, such that:

$$
\mathrm{z}+\mathrm{y}=\alpha^{2}, \quad \mathrm{z}-\mathrm{y}=\beta^{2} \quad \text { and } \mathrm{x}=\alpha \cdot \beta \text {. }
$$

From equalities (2.9) it follows that:

$$
\mathrm{x}=\alpha \cdot \beta, \quad \mathrm{y}=\frac{\alpha^{2}-\beta^{2}}{2}, \quad \mathrm{z}=\frac{\alpha^{2}+\beta^{2}}{2},
$$

which is a primitive solution of equation (A). If we note:

$\alpha+\beta=2 \cdot a \quad$ and

then, from equalities (2.10), we obtain equalities $(*)$.

$$
\alpha-\beta=2 \cdot b,
$$




$$
\left(a^{2}-b^{2}\right)^{2}+(2 \cdot a \cdot b)^{2}=\left(a^{2}-b^{2}\right)^{2} \quad(2.12)
$$

shows that the triplet in the statement is (indeed) the solution of the equation (A).

In addition, if:

$(\mathrm{x}, \mathrm{y}, \mathrm{z})=\mathrm{d} \geq 2$,

then $d$ divides the numbers $z+x$ and $z$-x; i.e. $d$ divides $2 \cdot a^{2}$ and $d$ divides $2 \cdot b^{2}$. Because $a$ and $b$ are prime numbers between them, it follows that:

$\mathrm{d}=2$.

So $\mathrm{a}^{2}+\mathrm{b}^{2}$ is an even number, what cannot be because $\mathrm{a}$ and $\mathrm{b}$ have different parities (if $\mathrm{a}$ and $\mathrm{b}$ are both odd, then equation (A) leads to a modulo impossibility 4). Therefore,

$\mathrm{d}=1$,

so the solution in the statement of the theorem is primitive.

Definition 2.6: A triplet $(x, y, z) \in N^{*} \times N^{*} \times N^{*}$ of form (*) is called Pythagorean triplet (or pitagoric).

The following remark are required here:

Remark 2.7: The primitive solutions of equation (A) can also be obtained from the uniqueness of the decomposition in primitive factors in the Gauss's integers ring $\mathbf{Z}[\boldsymbol{i}]$ :

$$
x+i y=(a+i b)^{2} \text {, }
$$

whence we obtain that:

$$
x=a^{2}-b^{2} \quad \text { and } y=2 \cdot a \cdot b \text {, }
$$

equalities which, are replaced in equality (A), leads us to the solution (*).

Of course, such an approach to solving equation (A) it is not for the vast majority of secondary school or high school students, but only for those high school students who know the relationship of divisibility in the ring $\mathbf{Z}[\mathbf{i}]$.

To list all primitive solutions of equation (A) we will consider for the number , $a$ " the values of $2,3,4, \ldots$ and then we will take those,$b$ " that are smaller than a, relative primes with a and different parities. For example:

$$
(a, b) \in\{(2,1),(3,2),(4,1),(4,3),(5,2),(5,4),(6,1,),(6,5),(7,2),(7,4),(7,6),(8,1),(8,3),(8,5),(8,7), \ldots\},
$$

and with these values, according to equalities $(*)$, we obtain that the primitive solutions of equation (A) are:

$(\mathrm{x}, \mathrm{y}, \mathrm{z}) \in\{(3,4,5),(5,12,13),(15,8,17),(7,24,25),(21,20,29),(9,40,41),(35,12,37),(11,60,61),(45,28,53),(33,56,65)$,

$(13,84,85),(63,16,65),(55,48,73),(39,80,89),(15,112,113), \ldots\}$.

Of course, according to Remark 2.4, primitive solutions are also triplets:

$(\mathrm{x}, \mathrm{y}, \mathrm{z}) \in\{(4,3,5),(12,5,13),(8,15,17),(24,7,25),(20,21,29),(40,9,41),(12,35,37),(60,11,61),(28,45,53),(56,33,65)$, $(84,13,85),(16,63,65),(48,55,73),(80,39,89),(112,15,113), \ldots\}$.

In addition, at any solution $(\mathrm{x}, \mathrm{y}, \mathrm{z}) \in \mathbf{N}^{*} \times \mathbf{N}^{*} \times \mathbf{N}^{*}$, we can associate another seven solutions from $\mathbf{Z} \times \mathbf{Z} \times \mathbf{Z}$, so determining a solution $(\mathrm{x}, \mathrm{y}, \mathrm{z}) \in \mathbf{N}^{*} \times \mathbf{N}^{*} \times \mathbf{N}^{*}$ means, in fact, the determination of eight solutions $( \pm x, \pm \mathrm{y}, \pm \mathrm{z}) \in \mathbf{Z} \times \mathbf{Z} \times \mathbf{Z}$, which can be expressed as follows:

Corollary 2.8: All solutions of equation ( $A$ ) are given by equalities:

$$
x=k \cdot\left(a^{2}-b^{2}\right), \quad y=2 \cdot k \cdot a \cdot b \quad \text { and } \quad z=k \cdot\left(a^{2}+b^{2}\right),
$$

where $k, a, b \in \mathbf{Z}$.

At the end of this paragraph we present the following immediate results:

Corollary 2.9:If $x, y, z \in \mathbf{Z}$, is the solution of equation $(A)$, then:

$$
a=x+2 \cdot y-2 \cdot z, \quad b=2 \cdot x+y-2 \cdot z, \quad \text { and } \quad c=2 \cdot x+2 \cdot y-3 \cdot z \text {, }
$$

is also the solution of the same equations.

Proof: A simple calculation shows us that:

$$
\begin{array}{ll}
a^{2}=x^{2}+4 \cdot y^{2}+4 \cdot z^{2}+4 \cdot x \cdot y-8 \cdot y \cdot z-4 \cdot x \cdot z, & b^{2}=4 \cdot x^{2}+y^{2}+4 \cdot z^{2}+4 \cdot x \cdot y-4 \cdot y \cdot z-8 \cdot x \cdot z \\
\text { and } & c^{2}=4 \cdot x^{2}+4 \cdot y^{2}+9 \cdot z^{2}+8 \cdot x \cdot y-12 \cdot y \cdot z-12 \cdot x \cdot z \cdot(2.13)
\end{array}
$$

Then, according to the hypothesis, from equalities (2.13), we obtain that:

$$
\begin{aligned}
\mathrm{a}^{2}+\mathrm{b}^{2} & =5 \cdot \mathrm{x}^{2}+5 \cdot \mathrm{y}^{2}+8 \cdot \mathrm{z}^{2}+8 \cdot \mathrm{x} \cdot \mathrm{y}-12 \cdot \mathrm{y} \cdot \mathrm{z}-12 \cdot \mathrm{x} \cdot \mathrm{z} \\
& =4 \cdot \mathrm{x}^{2}+4 \cdot \mathrm{y}^{2}+9 \cdot \mathrm{z}^{2}+8 \cdot \mathrm{x} \cdot \mathrm{y}-12 \cdot \mathrm{y} \cdot \mathrm{z}-12 \cdot \mathrm{x} \cdot \mathrm{z}+\left(\mathrm{x}^{2}+\mathrm{y}^{2}-\mathrm{z}^{2}\right) \\
& =\mathrm{c}^{2} .
\end{aligned}
$$

Remark 2.10: If $\left(x_{0}, y_{0}, z_{0}\right)$ is a primitive solution in nonzero natural numbers of equation $(A)$, then:

$$
3|(a \cdot b), \quad 4|(a \cdot b) \quad \text { and } \quad 5 \mid(a \cdot b \cdot c) \text {. (2.14) }
$$

Proof: Suppose that 3 does not divides either a and $b$. Then:

$$
\mathrm{a}=\mathscr{M}_{3} \pm 1 \quad \text { and } \mathrm{b}=\mathscr{M}_{3} \pm 1 \text {, }
$$

and, according to the hypothesis, 


$$
\mathrm{c}^{2}=\mathscr{M}_{3}+2
$$

which is impossible, because any perfect square, which is not a multiple of 3 , can only be $\mathscr{\mathscr { M }}_{3}+1$. Further, according to the hypothesis, from the proof of Theorem 2.5, it follows that one of the numbers $a$ or $b$ is even. Suppose $b$ is even, and a and c are odd, so:

$$
\mathrm{a}=2 \cdot \mathrm{m}+1, \quad \mathrm{~b}=2 \cdot \mathrm{n} \quad \mathrm{c}=2 \cdot \mathrm{p}+1, \quad \text { and } \quad \text { (2.16) }
$$

where $m . n, p \in \mathbf{N}^{*}$. Then the equality:

$$
a^{2}+b^{2}=c^{2}
$$

implies:

$$
m^{2}+m+n^{2}=p^{2}+p
$$

which shows that $\mathrm{n}$ is even, in which case 4 divides $\mathrm{b}$. Finally, suppose that 5 does not divide any of $\mathrm{a}, \mathrm{b}$ or $\mathrm{c}$ numbers. Then each of them is of the form $\mathscr{M}_{5} \pm 1$ or $\mathscr{M}_{5} \pm 2$, in which case their squares are only $\mathscr{M}_{5} \pm 1$ form. Now equality (A) leads us to a contradiction. Therefore, 5 divides at least one of the numbers $\mathrm{a}, \mathrm{b}$ or $\mathrm{c}$.

\section{Determination of the 2nd order Pythagorean matrices}

Of course, equation (A) admits solutions $(*)$ in any $\operatorname{ring}(\mathrm{R},+, \cdot)$, with $\mathrm{a}, \mathrm{b} \in \mathrm{R}$, when elements a and $\mathrm{b}$ commutes between them, i.e.:

$$
a \cdot b=b \cdot a \text {. }
$$

Moreover, it is known that the (commutative) field of complex numbers $(\mathbf{C},+, \cdot)$ is isomorphic to the field $(\mathrm{K},+, \cdot)$, where:

$$
\mathrm{K}=\left\{\left(\begin{array}{cc}
\mathrm{a} & \mathrm{b} \\
-\mathrm{b} & \mathrm{a}
\end{array}\right) \mid \mathrm{a}, \mathrm{b} \in \mathrm{R}\right\},
$$

and „,+, respectively „," are the addition or the usual multiplication of matrices. Therefore, any complex number (real, rational, integer or natural) can be represented as a matrix. For example, any number of real / rational / whole / natural may be written in the form:

$$
\left(\begin{array}{ll}
\mathrm{a} & 0 \\
0 & \mathrm{a}
\end{array}\right)=\mathrm{a} \cdot \mathrm{I}_{2}
$$

where $\mathrm{I}_{2} \in \mathrm{M}_{2}(\mathbf{C})$ is the identity matrix of the 2 nd order, that is:

$$
\mathrm{I}_{2}=\left(\begin{array}{ll}
1 & 0 \\
0 & 1
\end{array}\right) \text {. }
$$

In view of these issues, in this paragraph we will try to find solutions of equation $(\mathrm{A})$ in the ring $\left(\mathscr{M}_{2}(\mathbf{Z}),+, \cdot\right)$, replacing elements a and $\mathrm{b}$ with matrices $\mathrm{A}, \mathrm{B} \in \mathscr{\mathscr { M }}_{2}(\mathbf{N})$. Therefore, we consider the equation $(\mathrm{A})$ the set $\mathscr{M}_{2}(\mathbf{Z})$, equation that we write so:

$$
\mathrm{X}^{2}+\mathrm{Y}^{2}=\mathrm{Z}^{2}
$$

where $\mathrm{X}, \mathrm{Y}$ and $\mathrm{Z}$ are matrices of $\mathscr{\mathscr { M }}_{2}(\mathbf{Z})$ and let be matrices $\mathrm{A}, \mathrm{B} \in \mathscr{\mathscr { M }}_{2}(\mathbf{N})$, where:

$$
A=\left(\begin{array}{ll}
a & b \\
c & d
\end{array}\right) \quad \text { and } \quad B=\left(\begin{array}{cc}
x & y \\
z & t
\end{array}\right) \text {, }
$$

with $a, b, c, d, x, y, z, t \in \mathbf{N}$.

It is easily verified the next result:

Theorem 3.1: If the matrices $A$ and $B$ commutes between them, that is:

$$
A \cdot B=B \cdot A \text {, }
$$

then matrices:

$$
X=A^{2}-B^{2}, \quad Y=2 \cdot A \cdot B \quad \text { and } \quad Z=A^{2}+B^{2},
$$

are solutions of the equation ( $\left.A^{\prime \prime}\right)$.

Of course if:

$\mathrm{A}=\mathrm{B}=\mathrm{O}_{2}$,

then, according to equalities $(3,3)$, the matrices:

$\mathrm{X}=\mathrm{Y}=\mathrm{Z}=\mathrm{O}_{2}$

verify equation $\left(\mathrm{A}^{\prime \prime}\right)$. Also, if:

$\mathrm{A}=\mathrm{O}_{2}$, 
Then B can be any matrix of $\mathscr{\mathscr { M }}_{2}(\mathbf{N})$ and, thus, $\mathrm{X}, \mathrm{Y}, \mathrm{Z}$ - determined by the equalities (3.3), verify the equation $\left(\mathrm{A}^{\prime \prime}\right)$. The same and if:

$\mathrm{B}=\mathrm{O}_{2}$,

Then $\mathrm{A}$ can be any matrix of $\mathscr{M}_{2}(\mathbf{N})$ and, again, $\mathrm{X}, \mathrm{Y}$ and $\mathrm{Z}$ - determined by the same equalities (3.3), verify the equation $\left(\mathrm{A}^{\prime \prime}\right)$. Therefore, we will further consider that the $\mathrm{A}$ and $\mathrm{B}$ matrices are not null.

Then, from equalities (3.2), it follows that:

$$
\begin{array}{lll}
A^{2}=\left(\begin{array}{ll}
a^{2}+b \cdot c & b \cdot(a+d) \\
c \cdot(a+d) & d^{2}+b \cdot c
\end{array}\right) & \text { and } & B^{2}=\left(\begin{array}{ll}
x^{2}+y \cdot z & y \cdot(x+t) \\
z \cdot(x+t) & t^{2}+y \cdot z
\end{array}\right) \\
A \cdot B=\left(\begin{array}{ll}
a \cdot x+b \cdot z \cdot y+b \cdot t \\
c \cdot x+d \cdot z & c \cdot y+d \cdot t
\end{array}\right) & \text { and } & B \cdot A=\left(\begin{array}{cc}
a \cdot x+c \cdot y & b \cdot x+d \cdot y \\
a \cdot z+c \cdot t & b \cdot z+d \cdot t
\end{array}\right) .
\end{array}
$$

From the commutativity condition $\left(3.1^{\prime}\right)$ and the equalities (3.5) follow the system:

$$
\left\{\begin{array}{l}
a \cdot x+b \cdot z=a \cdot x+c \cdot y \\
a \cdot y+b \cdot t=b \cdot x+d \cdot y \\
c \cdot x+d \cdot z=a \cdot z+c \cdot t \\
c \cdot y+d \cdot t=b \cdot z+d \cdot t
\end{array}\right. \text {. }
$$

From the first or last equation of the system (3.6) it follows that:

$$
\mathrm{b} \cdot \mathrm{z}=\mathrm{c} \cdot \mathrm{y} \text {. }
$$

Solving our problem is reduced to solving the system (3.6), which implies solving the equation (3.7). Here, however, we will distinguish ten cases.

Case 1: $b=c=y=z=0$. In this case we have:

$$
\mathrm{A}=\left(\begin{array}{ll}
\mathrm{a} & 0 \\
0 & \mathrm{~d}
\end{array}\right) \quad \text { and } \quad \mathrm{B}=\left(\begin{array}{ll}
\mathrm{x} & 0 \\
0 & \mathrm{t}
\end{array}\right)
$$

with a, $\mathrm{d}, \mathrm{x}, \mathrm{t} \in \mathbf{N}$ and:

$$
\begin{array}{ll}
A^{2}=\left(\begin{array}{cc}
a^{2} & 0 \\
0 & d^{2}
\end{array}\right) & \text { and } \quad B^{2}=\left(\begin{array}{cc}
x^{2} & 0 \\
0 & t^{2}
\end{array}\right), \\
A \cdot B & =\left(\begin{array}{cc}
a \cdot x & 0 \\
0 & d \cdot t
\end{array}\right)=B \cdot A .
\end{array}
$$

Then, according to the equalities (3.3):

$$
\begin{aligned}
& X=\left(\begin{array}{cc}
a^{2}-x^{2} & 0 \\
0 & d^{2}-t^{2}
\end{array}\right), \quad Y=\left(\begin{array}{cc}
2 \cdot a \cdot x & 0 \\
0 & 2 \cdot d \cdot t
\end{array}\right), \quad Z=\left(\begin{array}{cc}
a^{2}+x^{2} & 0 \\
0 & d^{2}+t^{2}
\end{array}\right), \\
& X^{2}=\left(\begin{array}{cc}
\left(a^{2}-x^{2}\right)^{2} & 0 \\
0 & \left(d^{2}-t^{2}\right)^{2}
\end{array}\right), \quad Y^{2}=\left(\begin{array}{cc}
4 \cdot a^{2} \cdot x^{2} & 0 \\
0 & 4 \cdot d^{2} \cdot t^{2}
\end{array}\right), \quad Z^{2}=\left(\begin{array}{cc}
\left(a^{2}+x^{2}\right)^{2} & 0 \\
0 & \left(d^{2}+t^{2}\right)^{2}
\end{array}\right)
\end{aligned}
$$

And the equality $\left(\mathrm{A}^{\prime \prime}\right)$ is immediately verified.

Example 3.1.1: Let be the matrices:

$$
\mathrm{A}=\left(\begin{array}{ll}
4 & 0 \\
0 & 3
\end{array}\right) \quad \text { and } \quad \mathrm{B}=\left(\begin{array}{ll}
1 & 0 \\
0 & 2
\end{array}\right) .
$$

Then:

$$
\mathrm{A}^{2}=\left(\begin{array}{cc}
16 & 0 \\
0 & 9
\end{array}\right), \quad \mathrm{B}^{2}=\left(\begin{array}{ll}
1 & 0 \\
0 & 4
\end{array}\right) \quad \text { and } \quad \mathrm{A} \cdot \mathrm{B}=\left(\begin{array}{ll}
4 & 0 \\
0 & 6
\end{array}\right)=\mathrm{B} \cdot \mathrm{A} .
$$

Now, according to the equalities (3.3), we have:

$$
\begin{array}{lll}
X=\left(\begin{array}{cc}
15 & 0 \\
0 & 5
\end{array}\right), & Y=\left(\begin{array}{cc}
8 & 0 \\
0 & 12
\end{array}\right), & Z=\left(\begin{array}{cc}
17 & 0 \\
0 & 13
\end{array}\right), \\
X^{2}=\left(\begin{array}{cc}
225 & 0 \\
0 & 25
\end{array}\right), & Y^{2}=\left(\begin{array}{cc}
64 & 0 \\
0 & 144
\end{array}\right), & Z^{2}=\left(\begin{array}{cc}
289 & 0 \\
0 & 169
\end{array}\right),
\end{array}
$$


And the equality $\left(\mathrm{A}^{\prime \prime}\right)$ is immediately verified.

Example 3.1.2: Let be the matrices:

$$
\mathrm{A}=\left(\begin{array}{ll}
5 & 0 \\
0 & 9
\end{array}\right) \quad \text { and } \quad \mathrm{B}=\left(\begin{array}{ll}
3 & 0 \\
0 & 4
\end{array}\right) .
$$

Then:

$$
\mathrm{A}^{2}=\left(\begin{array}{cc}
25 & 0 \\
0 & 81
\end{array}\right) \quad \mathrm{B}^{2}=\left(\begin{array}{cc}
9 & 0 \\
0 & 16
\end{array}\right) \quad \text { and } \quad \mathrm{A} \cdot \mathrm{B}=\left(\begin{array}{cc}
15 & 0 \\
0 & 36
\end{array}\right)=\mathrm{B} \cdot \mathrm{A} .
$$

Now, according to the equalities (3.3), we have:

$$
\begin{array}{lll}
X=\left(\begin{array}{cc}
16 & 0 \\
0 & 65
\end{array}\right), & Y=\left(\begin{array}{cc}
30 & 0 \\
0 & 72
\end{array}\right), & Z=\left(\begin{array}{cc}
34 & 0 \\
0 & 97
\end{array}\right), \\
X^{2}=\left(\begin{array}{cc}
256 & 0 \\
0 & 4225
\end{array}\right), & Y^{2}=\left(\begin{array}{cc}
900 & 0 \\
0 & 5184
\end{array}\right), & Z^{2}=\left(\begin{array}{cc}
1156 & 0 \\
0 & 9409
\end{array}\right),
\end{array}
$$

And the equality $\left(\mathrm{A}^{\prime \prime}\right)$ is immediately verified.

Case 2: $b=c=y=0, z \neq 0$. In this case, from the third equation of the system (3.6), it follows that:

Then:

$$
\mathrm{d} \cdot \mathrm{z}=\mathrm{a} \cdot \mathrm{z}, \quad \text { i.e. } \mathrm{d}=\mathrm{a} \text {. }
$$

$$
\mathrm{A}=\left(\begin{array}{ll}
\mathrm{a} & 0 \\
0 & \mathrm{a}
\end{array}\right) \quad \text { and }
$$

With $a, x, z, t \in \mathbf{N}$ and:

$$
\begin{aligned}
& \mathrm{A}^{2}=\left(\begin{array}{cc}
\mathrm{a}^{2} & 0 \\
0 & \mathrm{a}^{2}
\end{array}\right) \quad \text { and } \quad \mathrm{B}^{2}=\left(\begin{array}{cc}
\mathrm{x}^{2} & 0 \\
\mathrm{z} \cdot(\mathrm{x}+\mathrm{t}) & \mathrm{t}^{2}
\end{array}\right) \\
& \mathrm{A} \cdot \mathrm{B}=\left(\begin{array}{cc}
\mathrm{a} \cdot \mathrm{x} & 0 \\
\mathrm{a} \cdot \mathrm{z} & \mathrm{a} \cdot \mathrm{t}
\end{array}\right)=\mathrm{B} \cdot \mathrm{A} .
\end{aligned}
$$

Now, according to the equalities (3.3), we have:

$$
\begin{array}{ll}
X=\left(\begin{array}{cc}
\mathrm{a}^{2}-\mathrm{x}^{2} & 0 \\
-\mathrm{z} \cdot(\mathrm{x}+\mathrm{t}) & \mathrm{a}^{2}-\mathrm{t}^{2}
\end{array}\right), & \mathrm{Y}=\left(\begin{array}{cc}
2 \cdot \mathrm{a} \cdot \mathrm{x} & 0 \\
2 \cdot \mathrm{a} \cdot \mathrm{z} & 2 \cdot \mathrm{a} \cdot \mathrm{t}
\end{array}\right), \\
\mathrm{X}^{2}=\left(\begin{array}{cc}
\left(\mathrm{a}^{2}-\mathrm{x}^{2}\right)^{2} & \mathrm{Z}=\left(\begin{array}{cc}
\mathrm{a}^{2}+\mathrm{x}^{2} & 0 \\
\mathrm{z} \cdot(\mathrm{x}+\mathrm{t}) & \mathrm{a}^{2}+\mathrm{t}^{2}
\end{array}\right), \\
-\mathrm{z} \cdot(\mathrm{x}+\mathrm{t}) \cdot\left(2 \cdot \mathrm{a}^{2}-\mathrm{x}^{2}-\mathrm{t}^{2}\right) & \left(\mathrm{a}^{2}-\mathrm{t}^{2}\right)^{2}
\end{array}\right), \\
\mathrm{Z}^{2}=\left(\begin{array}{cc}
\left(\mathrm{a}^{2}+\mathrm{x}^{2}\right) & 0 \\
\mathrm{z} \cdot(\mathrm{x}+\mathrm{t}) \cdot\left(2 \cdot \mathrm{a}^{2}+\mathrm{x}^{2}+\mathrm{t}^{2}\right) & \left(\mathrm{a}^{2}+\mathrm{t}^{2}\right)
\end{array}\right),
\end{array}
$$

And the equality $\left(\mathrm{A}^{\prime \prime}\right)$ is immediately verified.

Example 3.2.1: Let be the matrices:

$$
\mathrm{A}=\left(\begin{array}{ll}
4 & 0 \\
0 & 4
\end{array}\right) \quad \text { and } \mathrm{B}=\left(\begin{array}{ll}
1 & 0 \\
3 & 2
\end{array}\right) .
$$

Then:

$$
\mathrm{A}^{2}=\left(\begin{array}{cc}
16 & 0 \\
0 & 16
\end{array}\right), \quad \mathrm{B}^{2}=\left(\begin{array}{cc}
1 & 0 \\
9 & 4
\end{array}\right) \quad \mathrm{A} \cdot \mathrm{B}=\left(\begin{array}{cc}
4 & 0 \\
12 & 8
\end{array}\right)=\mathrm{B} \cdot \mathrm{A} \cdot
$$

Now, according to the equalities (3.3), we have:

$$
\begin{array}{lll}
X=\left(\begin{array}{cc}
15 & 0 \\
-9 & 12
\end{array}\right), & Y=\left(\begin{array}{cc}
8 & 0 \\
24 & 16
\end{array}\right), & Z=\left(\begin{array}{cc}
17 & 0 \\
9 & 20
\end{array}\right), \\
X^{2}=\left(\begin{array}{cc}
225 & 0 \\
-243 & 144
\end{array}\right), & Y^{2}=\left(\begin{array}{cc}
64 & 0 \\
576 & 256
\end{array}\right), & Z^{2}=\left(\begin{array}{cc}
289 & 0 \\
333 & 400
\end{array}\right),
\end{array}
$$

And the equality $\left(\mathrm{A}^{\prime \prime}\right)$ is immediately verified. 
Example 3.2.2: Let be the matrices:

$$
\mathrm{A}=\left(\begin{array}{ll}
5 & 0 \\
0 & 5
\end{array}\right) \quad \text { and } \quad \mathrm{B}=\left(\begin{array}{ll}
3 & 0 \\
7 & 4
\end{array}\right) .
$$

Then:

$$
\mathrm{A}^{2}=\left(\begin{array}{cc}
25 & 0 \\
0 & 25
\end{array}\right), \quad \mathrm{B}^{2}=\left(\begin{array}{cc}
9 & 0 \\
49 & 16
\end{array}\right) \quad \text { and } \quad \mathrm{A} \cdot \mathrm{B}=\left(\begin{array}{cc}
15 & 0 \\
35 & 20
\end{array}\right)=\mathrm{B} \cdot \mathrm{A}
$$

Now, according to the equalities (3.3), we have:

$$
\begin{array}{lll}
X=\left(\begin{array}{cc}
16 & 0 \\
-49 & 9
\end{array}\right), & Y=\left(\begin{array}{cc}
30 & 0 \\
70 & 40
\end{array}\right), & Z=\left(\begin{array}{ll}
34 & 0 \\
49 & 41
\end{array}\right), \\
X^{2}=\left(\begin{array}{cc}
256 & 0 \\
-1225 & 81
\end{array}\right), & Y^{2}=\left(\begin{array}{cc}
900 & 0 \\
4900 & 1600
\end{array}\right), & Z^{2}=\left(\begin{array}{cc}
1156 & 0 \\
3675 & 1681
\end{array}\right),
\end{array}
$$

And the equality $\left(\mathrm{A}^{\prime \prime}\right)$ is immediately verified.

Case 3: $b=c=z=0, y \neq 0$. In this case, from the second equation of the system (4), it follows that:

Then:

$$
\mathrm{a} \cdot \mathrm{y}=\mathrm{d} \cdot \mathrm{y}, \quad \text { i.e. } \mathrm{d}=\mathrm{a} \text {. }
$$

$$
\mathrm{A}=\left(\begin{array}{ll}
\mathrm{a} & 0 \\
0 & \mathrm{a}
\end{array}\right) \quad \text { and } \quad \mathrm{B}=\left(\begin{array}{ll}
\mathrm{x} & \mathrm{y} \\
0 & \mathrm{t}
\end{array}\right),
$$

With a, $x, y, t \in \mathbf{N}$ and:

$$
\begin{aligned}
& \mathrm{A}^{2}=\left(\begin{array}{cc}
\mathrm{a}^{2} & 0 \\
0 & \mathrm{a}^{2}
\end{array}\right)=\mathrm{a}^{2} \cdot \mathrm{I}_{2} \\
& \text { and } \\
& \mathrm{B}^{2}=\left(\begin{array}{cc}
\mathrm{x}^{2} & \mathrm{y} \cdot(\mathrm{x}+\mathrm{t}) \\
0 & \mathrm{t}^{2}
\end{array}\right), \\
& \mathrm{A} \cdot \mathrm{B}=\left(\begin{array}{cc}
\mathrm{a} \cdot \mathrm{x} & \mathrm{a} \cdot \mathrm{y} \\
0 & \mathrm{a} \cdot \mathrm{t}
\end{array}\right)=\mathrm{B} \cdot \mathrm{A} .
\end{aligned}
$$

Now, according to the equalities (3.3), we have:

$$
\begin{aligned}
& X=\left(\begin{array}{cc}
a^{2}-x^{2} & 0 \\
-z \cdot(x+t) & a^{2}-t^{2}
\end{array}\right), \quad Y=\left(\begin{array}{cc}
2 \cdot a \cdot x & 2 \cdot a \cdot y \\
0 & 2 \cdot a \cdot t
\end{array}\right), \quad Z=\left(\begin{array}{cc}
a^{2}+x^{2} & y \cdot(x+t) \\
0 & a^{2}+t^{2}
\end{array}\right), \\
& X^{2}=\left(\begin{array}{cc}
\left(a^{2}-x^{2}\right)^{2} & -z \cdot(x+t) \cdot\left(2 \cdot a^{2}-x^{2}-t^{2}\right) \\
0 & \left(a^{2}-t^{2}\right)^{2}
\end{array}\right), \quad Y^{2}=\left(\begin{array}{cc}
4 \cdot a^{2} \cdot x^{2} & 4 \cdot a^{2} \cdot y \cdot(x+t) \\
0 & 4 \cdot a^{2} \cdot t^{2}
\end{array}\right) \text {, } \\
& \mathrm{Z}^{2}=\left(\begin{array}{cc}
\left(\mathrm{a}^{2}+\mathrm{x}^{2}\right) & \mathrm{y} \cdot(\mathrm{x}+\mathrm{t}) \cdot\left(2 \cdot \mathrm{a}^{2}+\mathrm{x}^{2}+\mathrm{t}^{2}\right) \\
0 & \left(\mathrm{~d}^{2}+\mathrm{t}^{2}\right)
\end{array}\right),
\end{aligned}
$$

And the equality $\left(\mathrm{A}^{\prime \prime}\right)$ is immediately verified.

Example 3.3.1: Let be the matrices:

$$
\mathrm{A}=\left(\begin{array}{ll}
4 & 0 \\
0 & 4
\end{array}\right) \quad \text { and } \quad \mathrm{B}=\left(\begin{array}{ll}
1 & 3 \\
0 & 2
\end{array}\right) .
$$

Then:

$$
\mathrm{A}^{2}=\left(\begin{array}{cc}
16 & 0 \\
0 & 16
\end{array}\right), \quad \mathrm{B}^{2}=\left(\begin{array}{cc}
1 & 9 \\
0 & 4
\end{array}\right) \quad \text { and } \quad \mathrm{A} \cdot \mathrm{B}=\left(\begin{array}{cc}
4 & 12 \\
0 & 8
\end{array}\right)=\mathrm{B} \cdot \mathrm{A} \text {. }
$$

Now, according to the equalities (3.3), we have:

$$
\begin{array}{lll}
X=\left(\begin{array}{cc}
15 & -9 \\
0 & 12
\end{array}\right), & Y=\left(\begin{array}{ll}
8 & 24 \\
0 & 16
\end{array}\right), & Z=\left(\begin{array}{cc}
17 & 9 \\
0 & 20
\end{array}\right), \\
X^{2}=\left(\begin{array}{cc}
225 & -243 \\
0 & 144
\end{array}\right), & Y^{2}=\left(\begin{array}{cc}
64 & 576 \\
0 & 256
\end{array}\right), & Z^{2}=\left(\begin{array}{cc}
289 & 333 \\
0 & 400
\end{array}\right),
\end{array}
$$

And the equality $\left(\mathrm{A}^{\prime \prime}\right)$ is immediately verified.

Example 3.3.2: Let be the matrices: 


$$
\mathrm{A}=\left(\begin{array}{ll}
5 & 0 \\
0 & 5
\end{array}\right)
$$

and

$$
\mathrm{B}=\left(\begin{array}{ll}
3 & 7 \\
0 & 4
\end{array}\right)
$$

Then:

$$
\mathrm{A}^{2}=\left(\begin{array}{cc}
25 & 0 \\
0 & 25
\end{array}\right), \quad \mathrm{B}^{2}=\left(\begin{array}{cc}
9 & 49 \\
0 & 16
\end{array}\right) \quad \text { and } \quad \mathrm{A} \cdot \mathrm{B}=\left(\begin{array}{cc}
15 & 35 \\
0 & 20
\end{array}\right)=\mathrm{B} \cdot \mathrm{A} .
$$

Now, according to the equalities (3.3), we have:

$$
\begin{array}{lll}
X=\left(\begin{array}{cc}
16 & -49 \\
0 & 9
\end{array}\right), & Y=\left(\begin{array}{cc}
30 & 70 \\
0 & 40
\end{array}\right), & Z=\left(\begin{array}{cc}
34 & 49 \\
0 & 41
\end{array}\right), \\
X^{2}=\left(\begin{array}{cc}
256 & -1225 \\
0 & 81
\end{array}\right), & Y^{2}=\left(\begin{array}{cc}
900 & 4900 \\
0 & 1600
\end{array}\right), & Z^{2}=\left(\begin{array}{cc}
1156 & 3675 \\
0 & 1681
\end{array}\right),
\end{array}
$$

And the equality $\left(\mathrm{A}^{\prime \prime}\right)$ is immediately verified.

Case 4: $b=y=z=0, c \neq 0$. In this case, from the third equation of the system (3.6), it follows that:

Then:

$\mathrm{c} \cdot \mathrm{x}=\mathrm{c} \cdot \mathrm{t}$,

$$
A=\left(\begin{array}{ll}
a & 0 \\
c & d
\end{array}\right)
$$

and

With a, c, d, $\mathbf{x} \in \mathbf{N}$ and:

$$
\begin{aligned}
& A^{2}=\left(\begin{array}{cc}
a^{2} & 0 \\
c \cdot(a+d) & d^{2}
\end{array}\right) \\
& A \cdot B=\left(\begin{array}{cc}
a \cdot x & 0 \\
c \cdot x & d \cdot x
\end{array}\right)=B \cdot A .
\end{aligned}
$$

and $\mathrm{x}=\mathrm{t}$.

$$
\mathrm{B}=\left(\begin{array}{ll}
\mathrm{x} & 0 \\
0 & \mathrm{x}
\end{array}\right)
$$

Now, according to the equalities (3.3), we have:

$$
\begin{aligned}
& X=\left(\begin{array}{cc}
a^{2}-x^{2} & 0 \\
c \cdot(a+d) & d^{2}-x^{2}
\end{array}\right), \\
& X^{2}=\left(\begin{array}{cc}
\left(a^{2}-x^{2}\right)^{2} & Y=\left(\begin{array}{cc}
2 \cdot a \cdot x & 0 \\
2 \cdot c \cdot x & 2 \cdot d \cdot x
\end{array}\right), \\
c \cdot(a+d) \cdot\left(a^{2}+d^{2}-2 \cdot x^{2}\right) & \left(d^{2}-x^{2}\right)^{2}
\end{array}\right), \\
& Z^{2}=\left(\begin{array}{cc}
\left(a^{2}+x^{2}\right)^{2} & 0 \\
c \cdot(a+d) \cdot\left(a^{2}+d^{2}+2 \cdot x^{2}\right) & \left(d^{2}+x^{2}\right)^{2}
\end{array}\right),
\end{aligned}
$$$$
\mathrm{B}^{2}=\left(\begin{array}{cc}
\mathrm{x}^{2} & 0 \\
0 & \mathrm{x}^{2}
\end{array}\right)
$$

$Z=\left(\begin{array}{cc}a^{2}+x^{2} & 0 \\ c \cdot(a+d) & d^{2}+x^{2}\end{array}\right)$

And the equality $\left(\mathrm{A}^{\prime \prime}\right)$ is immediately verified.

Example 3.4.1: Let be the matrices:

$$
\mathrm{A}=\left(\begin{array}{ll}
4 & 0 \\
3 & 5
\end{array}\right) \quad \text { and } \quad \mathrm{B}=\left(\begin{array}{ll}
2 & 0 \\
0 & 2
\end{array}\right) .
$$

Then:

$$
\mathrm{A}^{2}=\left(\begin{array}{cc}
16 & 0 \\
27 & 25
\end{array}\right), \quad \mathrm{B}^{2}=\left(\begin{array}{cc}
4 & 0 \\
0 & 4
\end{array}\right) \quad \text { and } \quad \mathrm{A} \cdot \mathrm{B}=\left(\begin{array}{cc}
8 & 0 \\
6 & 10
\end{array}\right)=\mathrm{B} \cdot \mathrm{A} \cdot
$$

Now, according to the equalities (3.3), we have:

$$
\begin{array}{lll}
X=\left(\begin{array}{cc}
12 & 0 \\
27 & 21
\end{array}\right), & Y=\left(\begin{array}{cc}
16 & 0 \\
12 & 20
\end{array}\right), & Z=\left(\begin{array}{cc}
20 & 0 \\
27 & 29
\end{array}\right), \\
X^{2}=\left(\begin{array}{cc}
144 & 0 \\
891 & 441
\end{array}\right), & Y^{2}=\left(\begin{array}{cc}
256 & 0 \\
432 & 400
\end{array}\right), & Z^{2}=\left(\begin{array}{cc}
400 & 0 \\
1323 & 841
\end{array}\right),
\end{array}
$$

And the equality $\left(\mathrm{A}^{\prime \prime}\right)$ is immediately verified.

Example 3.4.2: Let be the matrices: 


$$
\mathrm{A}=\left(\begin{array}{ll}
6 & 0 \\
5 & 7
\end{array}\right) \quad \text { and } \quad \mathrm{B}=\left(\begin{array}{ll}
3 & 0 \\
0 & 3
\end{array}\right)
$$

Then:

$$
\mathrm{A}^{2}=\left(\begin{array}{cc}
36 & 0 \\
65 & 49
\end{array}\right), \quad \mathrm{B}^{2}=\left(\begin{array}{cc}
9 & 0 \\
0 & 9
\end{array}\right) \quad \text { and } \quad \mathrm{A} \cdot \mathrm{B}=\left(\begin{array}{cc}
18 & 0 \\
15 & 21
\end{array}\right)=\mathrm{B} \cdot \mathrm{A} .
$$

Now, according to the equalities (3.3), we have:

$$
\begin{array}{lll}
X=\left(\begin{array}{cc}
27 & 0 \\
65 & 40
\end{array}\right), & Y=\left(\begin{array}{cc}
36 & 0 \\
30 & 42
\end{array}\right), & Z=\left(\begin{array}{cc}
45 & 0 \\
65 & 58
\end{array}\right), \\
X^{2}=\left(\begin{array}{cc}
729 & 0 \\
4355 & 1600
\end{array}\right), & Y^{2}=\left(\begin{array}{cc}
1296 & 0 \\
2340 & 1764
\end{array}\right), & Z^{2}=\left(\begin{array}{cc}
2025 & 0 \\
6695 & 3364
\end{array}\right),
\end{array}
$$

And the equality $\left(\mathrm{A}^{\prime \prime}\right)$ is immediately verified.

Case 5: $c=y=z=0, b \neq 0$. In this case, from the second equation of the system (3.6), it follows that: $\mathrm{b} \cdot \mathrm{t}=\mathrm{b} \cdot \mathrm{x}$

Then:

$$
\mathrm{A}=\left(\begin{array}{ll}
\mathrm{a} & \mathrm{b} \\
0 & \mathrm{~d}
\end{array}\right) \quad \text { and }
$$

and

$$
\begin{aligned}
& A^{2}=\left(\begin{array}{cc}
a^{2} & b \cdot(a+d) \\
0 & d^{2}
\end{array}\right) \\
& A \cdot B=\left(\begin{array}{cc}
a \cdot x & b \cdot x \\
0 & d \cdot x
\end{array}\right)=B \cdot A .
\end{aligned}
$$

i.e.

$\mathrm{x}=\mathrm{t}$.

With $a, b, d, x \in \mathbf{N}$ and:

Now, according to the equalities (3.3), we have:

$$
\begin{aligned}
& X=\left(\begin{array}{cc}
a^{2}-x^{2} & b \cdot(a+d) \\
0 & d^{2}-x^{2}
\end{array}\right), \quad Y=\left(\begin{array}{cc}
2 \cdot a \cdot x & 2 \cdot b \cdot x \\
0 & 2 \cdot d \cdot x
\end{array}\right), \\
& X^{2}=\left(\begin{array}{cc}
\left(a^{2}-x^{2}\right)^{2} & b \cdot(a+d) \cdot\left(a^{2}+d^{2}-2 \cdot x^{2}\right) \\
0 & \left(d^{2}-x^{2}\right)^{2}
\end{array}\right), \\
& Z^{2}=\left(\begin{array}{cc}
\left(a^{2}+x^{2}\right)^{2} & b \cdot(a+d) \cdot\left(a^{2}+d^{2}+2 \cdot x^{2}\right) \\
0 & \left(d^{2}+x^{2}\right)^{2}
\end{array}\right),
\end{aligned}
$$

And the equality $\left(\mathrm{A}^{\prime \prime}\right)$ is immediately verified.

Example 3.5.1: Let be the matrices:

$$
\mathrm{A}=\left(\begin{array}{ll}
4 & 3 \\
0 & 5
\end{array}\right) \quad \text { and } \quad \mathrm{B}=\left(\begin{array}{ll}
2 & 0 \\
0 & 2
\end{array}\right) .
$$

Then:

$$
\mathrm{A}^{2}=\left(\begin{array}{cc}
16 & 27 \\
0 & 25
\end{array}\right), \quad \mathrm{B}^{2}=\left(\begin{array}{cc}
4 & 0 \\
0 & 4
\end{array}\right) \quad \text { and } \quad \mathrm{A} \cdot \mathrm{B}=\left(\begin{array}{cc}
8 & 6 \\
0 & 10
\end{array}\right)=\mathrm{B} \cdot \mathrm{A}
$$

Now, according to the equalities (3.3), we have:

$$
\begin{array}{lll}
X=\left(\begin{array}{cc}
12 & 27 \\
0 & 21
\end{array}\right), & Y=\left(\begin{array}{cc}
16 & 12 \\
0 & 20
\end{array}\right), & Z=\left(\begin{array}{cc}
20 & 27 \\
0 & 29
\end{array}\right), \\
X^{2}=\left(\begin{array}{cc}
144 & 891 \\
0 & 441
\end{array}\right), & Y^{2}=\left(\begin{array}{cc}
256 & 432 \\
0 & 400
\end{array}\right), & Z^{2}=\left(\begin{array}{cc}
400 & 1323 \\
0 & 841
\end{array}\right),
\end{array}
$$

And the equality $\left(\mathrm{A}^{\prime \prime}\right)$ is immediately verified.

Example 3.5.2: Let be the matrices: 


$$
\mathrm{A}=\left(\begin{array}{ll}
6 & 5 \\
0 & 7
\end{array}\right) \quad \text { and } \quad \mathrm{B}=\left(\begin{array}{ll}
3 & 0 \\
0 & 3
\end{array}\right)
$$

Then:

$$
\mathrm{A}^{2}=\left(\begin{array}{cc}
36 & 65 \\
0 & 49
\end{array}\right), \quad \mathrm{B}^{2}=\left(\begin{array}{cc}
9 & 0 \\
0 & 9
\end{array}\right) \quad \text { and } \quad \mathrm{A} \cdot \mathrm{B}=\left(\begin{array}{cc}
18 & 15 \\
0 & 21
\end{array}\right)=\mathrm{B} \cdot \mathrm{A} .
$$

Now, according to the equalities (3.3), we have:
$X\left(\begin{array}{cc}27 & 65 \\ 0 & 40\end{array}\right)$
$\mathrm{Y}=\left(\begin{array}{cc}36 & 30 \\ 0 & 42\end{array}\right)$,
$\mathrm{Z}=\left(\begin{array}{cc}45 & 65 \\ 0 & 58\end{array}\right)$,
$X^{2}=\left(\begin{array}{cc}729 & 4355 \\ 0 & 1600\end{array}\right)$,
$\mathrm{Y}^{2}=\left(\begin{array}{cc}1296 & 2340 \\ 0 & 1764\end{array}\right)$,
$Z^{2}=\left(\begin{array}{cc}2025 & 6695 \\ 0 & 3364\end{array}\right)$

And the equality $\left(\mathrm{A}^{\prime \prime}\right)$ is immediately verified.

Case 6: $b=c=0, y \neq 0, z \neq 0$. In this case, from the second equation of the system (3.6), it follows that:

Then:

$$
\mathrm{a} \cdot \mathrm{y}=\mathrm{d} \cdot \mathrm{y}, \quad \text { i.e. } \mathrm{d}=\mathrm{a} \text {. }
$$

$$
\mathrm{A}=\left(\begin{array}{ll}
\mathrm{a} & 0 \\
0 & \mathrm{a}
\end{array}\right) \quad \text { and } \quad \mathrm{B}=\left(\begin{array}{cc}
\mathrm{x} & \mathrm{y} \\
\mathrm{z} & \mathrm{t}
\end{array}\right),
$$

With a, $\mathrm{x}, \mathrm{y}, \mathrm{z}, \mathrm{t} \in \mathbf{N}$ and:

$$
\begin{aligned}
& A^{2}=\left(\begin{array}{cc}
a^{2} & 0 \\
0 & a^{2}
\end{array}\right) \quad \text { and } \quad B^{2}=\left(\begin{array}{cc}
x^{2}+y \cdot z & y \cdot(x+t) \\
z \cdot(x+t) & t^{2}+y \cdot z
\end{array}\right) \\
& A \cdot B=\left(\begin{array}{cc}
a \cdot x & a \cdot y \\
a \cdot z & a \cdot t
\end{array}\right)=B \cdot A .
\end{aligned}
$$

Now, according to the equalities (3.3), we have:

$$
\begin{aligned}
& X=\left(\begin{array}{cc}
a^{2}-x^{2}-y \cdot z & -y \cdot(x+t) \\
-z \cdot(x+t) & a^{2}-t^{2}-y \cdot z
\end{array}\right), \quad Y=\left(\begin{array}{cc}
2 \cdot a \cdot x & 2 \cdot a \cdot y \\
2 \cdot a \cdot z & 2 \cdot a \cdot t
\end{array}\right), \\
& Z=\left(\begin{array}{cc}
a^{2}+x^{2}+y \cdot z & y \cdot(x+t) \\
z \cdot(x+t) & a^{2}+t^{2}+y \cdot z
\end{array}\right), \\
& X^{2}=\left(\begin{array}{cc}
\left(a^{2}-x^{2}-y \cdot z\right)^{2}+y \cdot z \cdot(x+t)^{2} & -y \cdot(x+t) \cdot\left(2 \cdot a^{2}-x^{2}-t^{2}-2 \cdot y \cdot z\right) \\
-z \cdot(x+t) \cdot\left(2 \cdot a^{2}-x^{2}-t^{2}-2 \cdot y \cdot z\right) & \left(a^{2}-t^{2}-y \cdot z\right)^{2}+y \cdot z \cdot(x+t)^{2}
\end{array}\right), \\
& Y^{2}=\left(\begin{array}{cc}
4 \cdot a^{2} \cdot\left(x^{2}+y \cdot z\right) & 4 \cdot a^{2} \cdot y \cdot(x+t) \\
4 \cdot a^{2} \cdot z \cdot(x+t) & 4 \cdot a^{2} \cdot\left(t^{2}+y \cdot z\right)
\end{array}\right), \\
& Z^{2}=\left(\begin{array}{cc}
\left(a^{2}+x^{2}+y \cdot z\right)^{2}+y \cdot z \cdot(x+t)^{2} & y \cdot(x+t) \cdot\left(2 \cdot a^{2}+x^{2}+t^{2}+2 \cdot y \cdot z\right) \\
z \cdot(x+t) \cdot\left(2 \cdot a^{2}+x^{2}+t^{2}+2 \cdot y \cdot z\right) & \left(a^{2}+t^{2}+y \cdot z\right)^{2}+y \cdot z \cdot(x+t)^{2}
\end{array}\right)
\end{aligned}
$$

And the equality $\left(\mathrm{A}^{\prime \prime}\right)$ is immediately verified.

Example 3.6.1: Let be the matrices:

$$
\mathrm{A}=\left(\begin{array}{ll}
4 & 0 \\
0 & 4
\end{array}\right) \quad \text { and } \quad \mathrm{B}=\left(\begin{array}{ll}
1 & 3 \\
5 & 2
\end{array}\right) .
$$

Then:

$$
\mathrm{A}^{2}=\left(\begin{array}{cc}
16 & 0 \\
0 & 16
\end{array}\right), \quad \mathrm{B}^{2}=\left(\begin{array}{cc}
1 & 9 \\
15 & 4
\end{array}\right) \quad \text { and } \quad \mathrm{A} \cdot \mathrm{B}=\left(\begin{array}{cc}
4 & 12 \\
20 & 8
\end{array}\right)=\mathrm{B} \cdot \mathrm{A} .
$$

Now, according to the equalities (3.3), we have:

$$
X=\left(\begin{array}{cc}
15 & -9 \\
-15 & 12
\end{array}\right), \quad Y=\left(\begin{array}{cc}
8 & 24 \\
40 & 16
\end{array}\right), \quad Z=\left(\begin{array}{cc}
17 & 9 \\
15 & 20
\end{array}\right),
$$




$$
\mathrm{X}^{2}=\left(\begin{array}{cc}
225 & -243 \\
-405 & 144
\end{array}\right), \quad \mathrm{Y}^{2}=\left(\begin{array}{cc}
64 & 576 \\
960 & 256
\end{array}\right), \quad \mathrm{Z}^{2}=\left(\begin{array}{cc}
289 & 333 \\
555 & 400
\end{array}\right),
$$

And the equality $\left(\mathrm{A}^{\prime \prime}\right)$ is immediately verified.

Example 3.6.2: Let be the matrices:

$$
\mathrm{A}=\left(\begin{array}{ll}
5 & 0 \\
0 & 5
\end{array}\right) \quad \text { and } \quad \mathrm{B}=\left(\begin{array}{ll}
3 & 7 \\
5 & 4
\end{array}\right) .
$$

Then:

$$
\mathrm{A}^{2}=\left(\begin{array}{cc}
25 & 0 \\
0 & 25
\end{array}\right), \quad \mathrm{B}^{2}=\left(\begin{array}{cc}
9 & 49 \\
35 & 16
\end{array}\right) \quad \text { and } \quad \mathrm{A} \cdot \mathrm{B}=\left(\begin{array}{cc}
15 & 35 \\
25 & 20
\end{array}\right)=\mathrm{B} \cdot \mathrm{A}
$$

Now, according to the equalities (3.3), we have:

$$
\begin{array}{lll}
X=\left(\begin{array}{cc}
16 & -49 \\
35 & 9
\end{array}\right), & Y=\left(\begin{array}{ll}
30 & 70 \\
50 & 40
\end{array}\right), & Z=\left(\begin{array}{ll}
34 & 49 \\
35 & 41
\end{array}\right), \\
X^{2}=\left(\begin{array}{cc}
256 & -1225 \\
875 & 81
\end{array}\right), & Y^{2}=\left(\begin{array}{cc}
900 & 4900 \\
3500 & 1600
\end{array}\right), & Z^{2}=\left(\begin{array}{ll}
1156 & 3675 \\
2625 & 1681
\end{array}\right),
\end{array}
$$

And the equality $\left(\mathrm{A}^{\prime \prime}\right)$ is immediately verified.

Case 7: $b=y=0, c \neq 0, z \neq 0$. In this case, from the third equation of the system (3.6), it follows that:

$$
\mathrm{c} \cdot \mathrm{x}+\mathrm{d} \cdot \mathrm{z}=\mathrm{a} \cdot \mathrm{z}+\mathrm{c} \cdot \mathrm{t} \text {, }
$$

We distinguish two subcases:

$\alpha)$ If $\mathrm{a}=\mathrm{d}$, then (also) $\mathrm{x}=\mathrm{t}$. It follows that:

$$
\mathrm{A}=\left(\begin{array}{cc}
\mathrm{a} & 0 \\
\mathrm{c} & \mathrm{a}
\end{array}\right) \quad \text { and } \quad \mathrm{B}=\left(\begin{array}{cc}
\mathrm{x} & 0 \\
\mathrm{z} & \mathrm{x}
\end{array}\right) \text {, }
$$

With a, c, $x, z \in \mathbf{N}$ and:

$$
\begin{aligned}
& A^{2}=\left(\begin{array}{cc}
a^{2} & 0 \\
2 \cdot a \cdot c & a^{2}
\end{array}\right) \\
& A \cdot B=\left(\begin{array}{cc}
a \cdot x & 0 \\
a \cdot z+c \cdot x & a \cdot x
\end{array}\right)=B \cdot A .
\end{aligned}
$$$$
\text { and }
$$

i.e.

$$
\mathrm{z} \cdot(\mathrm{a}-\mathrm{d})=\mathrm{c} \cdot(\mathrm{x}-\mathrm{t}) \text {. }
$$

Now, according to the equalities (3.3), we have:

$$
\begin{aligned}
& X=\left(\begin{array}{cc}
a^{2}-x^{2} & 0 \\
2 \cdot(a \cdot c-x \cdot z) & a^{2}-x^{2}
\end{array}\right), \\
& Z=\left(\begin{array}{cc}
a^{2}+x^{2} & 0 \\
2 \cdot(a \cdot c+x \cdot z) & a^{2}+x^{2}
\end{array}\right), \\
& X^{2}=\left(\begin{array}{cc}
\left(a^{2}-x^{2}\right)^{2} & 0 \\
4 \cdot(a \cdot c-x \cdot z) \cdot\left(a^{2}-x^{2}\right) & \left(a^{2}-x^{2}\right)^{2}
\end{array}\right), \\
& Z^{2}=\left(\begin{array}{cc}
\left(a^{2}+x^{2}\right) & 0 \\
4 \cdot(a \cdot c+x \cdot z) \cdot\left(a^{2}+x^{2}\right) & \left(a^{2}+x^{2}\right)
\end{array}\right),
\end{aligned}
$$$$
\mathrm{Y}=\left(\begin{array}{cc}
2 \cdot \mathrm{a} \cdot \mathrm{x} & 0 \\
2 \cdot(\mathrm{a} \cdot \mathrm{z}+\mathrm{c} \cdot \mathrm{x}) & 2 \cdot \mathrm{a} \cdot \mathrm{x}
\end{array}\right)
$$

$$
Y^{2}=\left(\begin{array}{cc}
4 \cdot a^{2} \cdot x^{2} & 0 \\
8 \cdot a \cdot x \cdot(a \cdot z+c \cdot x) & 4 \cdot a^{2} \cdot x^{2}
\end{array}\right)
$$

And the equality $\left(\mathrm{A}^{\prime \prime}\right)$ is immediately verified.

Example 3.7.1: Let be the matrices:

$$
\mathrm{A}=\left(\begin{array}{ll}
4 & 0 \\
5 & 4
\end{array}\right) \quad \text { and } \quad \mathrm{B}=\left(\begin{array}{ll}
1 & 0 \\
3 & 1
\end{array}\right) .
$$

Then:

$$
\mathrm{A}^{2}=\left(\begin{array}{cc}
16 & 0 \\
40 & 16
\end{array}\right), \quad \mathrm{B}^{2}=\left(\begin{array}{ll}
1 & 0 \\
6 & 1
\end{array}\right)
$$

and

$$
\mathrm{A} \cdot \mathrm{B}=\left(\begin{array}{cc}
4 & 0 \\
17 & 4
\end{array}\right)=\mathrm{B} \cdot \mathrm{A}
$$

Now, according to the equalities (3.3), we have: 


$$
\begin{array}{lll}
X=\left(\begin{array}{cc}
15 & 0 \\
34 & 15
\end{array}\right), & Y=\left(\begin{array}{cc}
8 & 0 \\
34 & 8
\end{array}\right), & Z=\left(\begin{array}{cc}
17 & 0 \\
46 & 17
\end{array}\right), \\
X^{2}=\left(\begin{array}{cc}
225 & 0 \\
1020 & 225
\end{array}\right), & Y^{2}=\left(\begin{array}{cc}
64 & 0 \\
544 & 64
\end{array}\right), & Z^{2}=\left(\begin{array}{cc}
289 & 0 \\
1564 & 289
\end{array}\right),
\end{array}
$$

And the equality $\left(\mathrm{A}^{\prime \prime}\right)$ is immediately verified.

Example 3.7.2: Let be the matrices:

$$
\mathrm{A}=\left(\begin{array}{ll}
5 & 0 \\
2 & 5
\end{array}\right) \quad \text { and } \quad \mathrm{B}=\left(\begin{array}{ll}
3 & 0 \\
7 & 3
\end{array}\right) .
$$

Then:

$$
\mathrm{A}^{2}=\left(\begin{array}{cc}
25 & 0 \\
20 & 25
\end{array}\right), \quad \mathrm{B}^{2}=\left(\begin{array}{cc}
9 & 0 \\
42 & 9
\end{array}\right) \quad \text { and } \quad \mathrm{A} \cdot \mathrm{B}=\left(\begin{array}{cc}
15 & 0 \\
41 & 15
\end{array}\right)=\mathrm{B} \cdot \mathrm{A} .
$$

Now, according to the equalities (3.3), we have:

$$
\begin{array}{lll}
X=\left(\begin{array}{cc}
16 & 0 \\
-22 & 16
\end{array}\right), & Y=\left(\begin{array}{cc}
30 & 0 \\
82 & 30
\end{array}\right), & Z=\left(\begin{array}{ll}
34 & 0 \\
62 & 34
\end{array}\right), \\
X^{2}=\left(\begin{array}{cc}
256 & 0 \\
-704 & 256
\end{array}\right), & Y^{2}=\left(\begin{array}{cc}
900 & 0 \\
4920 & 900
\end{array}\right), & Z^{2}=\left(\begin{array}{cc}
1156 & 0 \\
4216 & 1156
\end{array}\right),
\end{array}
$$

And the equality $\left(\mathrm{A}^{\prime \prime}\right)$ is immediately verified.

$\beta$ ) If $a \neq d$, then (also) $x \neq t$. It follows that:

$$
\mathrm{z}=\frac{\mathrm{c} \cdot(\mathrm{x}-\mathrm{t})}{\mathrm{a}-\mathrm{d}}
$$

with

$(a-d) \mid b \cdot(x-t)$

And

$$
A=\left(\begin{array}{ll}
a & 0 \\
c & d
\end{array}\right) \quad \text { and } \quad B=\left(\begin{array}{cc}
x & 0 \\
\frac{c \cdot(x-t)}{a-d} & t
\end{array}\right) \text {, }
$$

With a, c, d, $x, t \in \mathbf{N}, \frac{\mathrm{x}-\mathrm{t}}{\mathrm{a}-\mathrm{d}}>0$ and:

$$
\begin{aligned}
& A^{2}=\left(\begin{array}{cc}
a^{2} & 0 \\
c \cdot(a+d) & d^{2}
\end{array}\right) \\
& \mathrm{A} \cdot \mathrm{B}=\left(\begin{array}{cc}
\mathrm{a} \cdot \mathrm{x} & 0 \\
\frac{\mathrm{c} \cdot(\mathrm{a} \cdot \mathrm{x}-\mathrm{d} \cdot \mathrm{t})}{\mathrm{a}-\mathrm{d}} & \mathrm{d} \cdot \mathrm{t}
\end{array}\right)=\mathrm{B} \cdot \mathrm{A} .
\end{aligned}
$$$$
\text { and }
$$$$
\mathrm{B}^{2}=\left(\begin{array}{cc}
\mathrm{x}^{2} & 0 \\
\frac{\mathrm{c} \cdot\left(\mathrm{x}^{2}-\mathrm{t}^{2}\right)}{\mathrm{a}-\mathrm{d}} & \mathrm{t}^{2}
\end{array}\right)
$$

Now, according to the equalities (3.3), we have:

$$
\begin{aligned}
& X=\left(\begin{array}{cc}
\frac{c \cdot\left(a^{2}-d^{2}-x^{2}+t^{2}\right)}{a-d} & d^{2}-t^{2}
\end{array}\right), \\
& Z=\left(\begin{array}{cc}
\frac{a^{2}+x^{2}}{c \cdot\left(a^{2}-d^{2}+x^{2}-t^{2}\right)} & 0 \\
a-d & d^{2}+t^{2}
\end{array}\right), \\
& X^{2}=\left(\begin{array}{cc}
\frac{c \cdot\left(a^{2}-d^{2}-x^{2}+t^{2}\right) \cdot\left(a^{2}+d^{2}-x^{2}-t^{2}\right)}{a-d} & \left(d^{2}-t^{2}\right)^{2}
\end{array}\right), \\
& Y^{2}=\left(\begin{array}{cc}
\frac{4 \cdot c \cdot\left(a^{2} \cdot x^{2}-d^{2} \cdot t^{2}\right)}{a-d} & 4 \cdot d^{2} \cdot t^{2}
\end{array}\right),
\end{aligned}
$$

$$
\mathrm{Y}=\left(\begin{array}{cc}
2 \cdot \mathrm{a} \cdot \mathrm{x} \cdot(\mathrm{a} \cdot \mathrm{x}-\mathrm{d} \cdot \mathrm{t}) & 0 \\
\mathrm{a}-\mathrm{d} & 2 \cdot \mathrm{d} \cdot \mathrm{t}
\end{array}\right)
$$




$$
\mathrm{Z}^{2}=\left(\begin{array}{cc}
\left(\mathrm{a}^{2}+\mathrm{x}^{2}\right)^{2} & 0 \\
\frac{\mathrm{c} \cdot\left(\mathrm{a}^{2}-\mathrm{d}^{2}+\mathrm{x}^{2}-\mathrm{t}^{2}\right) \cdot\left(\mathrm{a}^{2}+\mathrm{d}^{2}+\mathrm{x}^{2}+\mathrm{t}^{2}\right)}{\mathrm{a}-\mathrm{d}} & \left(\mathrm{d}^{2}+\mathrm{t}^{2}\right)^{2}
\end{array}\right),
$$

And the equality $\left(\mathrm{A}^{\prime \prime}\right)$ is immediately verified.

Example 3.7.3: Let be the matrices:

$$
\mathrm{A}=\left(\begin{array}{ll}
4 & 0 \\
5 & 3
\end{array}\right) \quad \text { and } \quad \mathrm{B}=\left(\begin{array}{ll}
2 & 0 \\
5 & 1
\end{array}\right) .
$$

Then:

$$
\mathrm{A}^{2}=\left(\begin{array}{ll}
16 & 0 \\
35 & 9
\end{array}\right), \quad \mathrm{B}^{2}=\left(\begin{array}{cc}
4 & 0 \\
15 & 1
\end{array}\right) \quad \text { and } \quad \mathrm{A} \cdot \mathrm{B}=\left(\begin{array}{cc}
8 & 0 \\
25 & 3
\end{array}\right)=\mathrm{B} \cdot \mathrm{A} .
$$

Now, according to the equalities (3.3), we have:

$$
\begin{array}{lll}
X=\left(\begin{array}{ll}
12 & 0 \\
20 & 8
\end{array}\right), & Y=\left(\begin{array}{cc}
16 & 0 \\
50 & 6
\end{array}\right), & Z=\left(\begin{array}{cc}
20 & 0 \\
50 & 10
\end{array}\right), \\
X^{2}=\left(\begin{array}{cc}
144 & 0 \\
400 & 64
\end{array}\right), & Y^{2}=\left(\begin{array}{cc}
256 & 0 \\
1100 & 36
\end{array}\right), & Z^{2}=\left(\begin{array}{cc}
400 & 0 \\
1100 & 100
\end{array}\right),
\end{array}
$$

And the equality $\left(\mathrm{A}^{\prime \prime}\right)$ is immediately verified.

Example 3.7.4: Let be the matrices:

$$
\mathrm{A}=\left(\begin{array}{ll}
5 & 0 \\
2 & 3
\end{array}\right) \quad \text { and } \quad \mathrm{B}=\left(\begin{array}{ll}
9 & 0 \\
7 & 2
\end{array}\right) .
$$

Then:

$$
\mathrm{A}^{2}=\left(\begin{array}{ll}
25 & 0 \\
16 & 9
\end{array}\right), \quad \mathrm{B}^{2}=\left(\begin{array}{ll}
81 & 0 \\
77 & 4
\end{array}\right) \quad \text { and } \quad \mathrm{A} \cdot \mathrm{B}=\left(\begin{array}{ll}
45 & 0 \\
39 & 6
\end{array}\right)=\mathrm{B} \cdot \mathrm{A} .
$$

Now, according to the equalities (3.3), we have:

$$
\begin{array}{lll}
X=\left(\begin{array}{ll}
-56 & 0 \\
-61 & 5
\end{array}\right), & Y=\left(\begin{array}{cc}
90 & 0 \\
78 & 12
\end{array}\right), & Z=\left(\begin{array}{cc}
106 & 0 \\
93 & 13
\end{array}\right), \\
X^{2}=\left(\begin{array}{cc}
3136 & 0 \\
3111 & 25
\end{array}\right), & Y^{2}=\left(\begin{array}{cc}
8100 & 0 \\
7956 & 144
\end{array}\right), & Z^{2}=\left(\begin{array}{ccc}
11236 & 0 \\
11067 & 169
\end{array}\right),
\end{array}
$$

And the equality $\left(\mathrm{A}^{\prime \prime}\right)$ is immediately verified.

Case 8: $c=z=0, b \neq 0, y \neq 0$. In this case, from the second equation of the system (4), it follows that:

$\mathrm{a} \cdot \mathrm{y}+\mathrm{b} \cdot \mathrm{t}=\mathrm{b} \cdot \mathrm{x}+\mathrm{d} \cdot \mathrm{y}$,

We also distinguish two subcases here:

$\alpha)$ If $\mathrm{a}=\mathrm{d}$, then (also) $\mathrm{x}=\mathrm{t}$. It follows that:

$$
\mathrm{A}=\left(\begin{array}{cc}
\mathrm{a} & \mathrm{b} \\
0 & \mathrm{a}
\end{array}\right) \quad \mathrm{B}=\left(\begin{array}{cc}
\mathrm{x} & \mathrm{y} \\
0 & \mathrm{x}
\end{array}\right),
$$

with $a, b, x, y \in \mathbf{N}$ and:

$$
\begin{aligned}
& A^{2}=\left(\begin{array}{cc}
a^{2} & 2 \cdot a \cdot b \\
0 & a^{2}
\end{array}\right) \\
& A \cdot B=\left(\begin{array}{cc}
a \cdot x & a \cdot y+b \cdot x \\
0 & a \cdot x
\end{array}\right)=B \cdot A .
\end{aligned}
$$$$
\text { and }
$$$$
\text { i.e. } \quad \mathrm{y} \cdot(\mathrm{a}-\mathrm{d})=\mathrm{b} \cdot(\mathrm{x}-\mathrm{t}) \text {. }
$$

Now, according to the equalities (3.3), we have:

$$
\begin{aligned}
& X=\left(\begin{array}{cc}
a^{2}-x^{2} & 2 \cdot(a \cdot b-x \cdot y) \\
0 & a^{2}-x^{2}
\end{array}\right), \\
& Z=\left(\begin{array}{cc}
a^{2}+x^{2} & 2 \cdot(a \cdot b+x \cdot y) \\
0 & a^{2}+x^{2}
\end{array}\right),
\end{aligned}
$$$$
Y=\left(\begin{array}{cc}
2 \cdot a \cdot x & 2 \cdot(a \cdot y+b \cdot x) \\
0 & 2 \cdot a \cdot x
\end{array}\right)
$$ 


$$
\begin{aligned}
& X^{2}=\left(\begin{array}{cc}
\left(a^{2}-x^{2}\right)^{2} & 4 \cdot(a \cdot b-x \cdot y) \cdot\left(a^{2}-x^{2}\right) \\
0 & \left(a^{2}-x^{2}\right)^{2}
\end{array}\right), \\
& Z^{2}=\left(\begin{array}{cc}
\left(a^{2}+x^{2}\right) & 4 \cdot(a \cdot b+x \cdot y) \cdot\left(a^{2}+x^{2}\right) \\
0 & \left(a^{2}+x^{2}\right)
\end{array}\right),
\end{aligned}
$$$$
Y^{2}=\left(\begin{array}{cc}
4 \cdot a^{2} \cdot x^{2} & 8 \cdot a \cdot x \cdot(a \cdot y+b \cdot x) \\
0 & 4 \cdot a^{2} \cdot x^{2}
\end{array}\right)
$$

and the equality $\left(\mathrm{A}^{\prime \prime}\right)$ is immediately verified.

Example 3.8.1: Let be the matrices:

$$
\mathrm{A}=\left(\begin{array}{ll}
4 & 5 \\
0 & 4
\end{array}\right) \quad \text { and } \quad \mathrm{B}=\left(\begin{array}{ll}
1 & 3 \\
0 & 1
\end{array}\right) .
$$

Then:

$$
\mathrm{A}^{2}=\left(\begin{array}{cc}
16 & 40 \\
0 & 16
\end{array}\right), \quad \mathrm{B}^{2}=\left(\begin{array}{cc}
1 & 6 \\
0 & 1
\end{array}\right) \quad \mathrm{A} \cdot \mathrm{B}=\left(\begin{array}{cc}
4 & 17 \\
0 & 4
\end{array}\right)=\mathrm{B} \cdot \mathrm{A} \text {. }
$$

Now, according to the equalities (3.3), we have:

$$
\begin{array}{lll}
X=\left(\begin{array}{cc}
15 & 34 \\
0 & 15
\end{array}\right), & Y=\left(\begin{array}{cc}
8 & 34 \\
0 & 8
\end{array}\right), & Z=\left(\begin{array}{cc}
17 & 46 \\
0 & 17
\end{array}\right), \\
X^{2}=\left(\begin{array}{cc}
225 & 1020 \\
0 & 225
\end{array}\right), & Y^{2}=\left(\begin{array}{cc}
64 & 544 \\
0 & 64
\end{array}\right), & Z^{2}=\left(\begin{array}{cc}
289 & 1564 \\
0 & 289
\end{array}\right),
\end{array}
$$

And the equality $\left(\mathrm{A}^{\prime \prime}\right)$ is immediately verified.

Example 3.8.2: Let be the matrices:

$$
\mathrm{A}=\left(\begin{array}{ll}
5 & 2 \\
0 & 5
\end{array}\right) \quad \text { and } \quad \mathrm{B}=\left(\begin{array}{ll}
3 & 7 \\
0 & 3
\end{array}\right) .
$$

Then:

$$
\mathrm{A}^{2}=\left(\begin{array}{cc}
25 & 20 \\
0 & 25
\end{array}\right), \quad \mathrm{B}^{2}=\left(\begin{array}{cc}
9 & 42 \\
0 & 9
\end{array}\right) \quad \text { and } \quad \mathrm{A} \cdot \mathrm{B}=\left(\begin{array}{cc}
15 & 41 \\
0 & 15
\end{array}\right)=\mathrm{B} \cdot \mathrm{A} \text {. }
$$

Now, according to the equalities (3.3), we have:

$$
\begin{aligned}
& X=\left(\begin{array}{cc}
16 & -22 \\
0 & 16
\end{array}\right), \\
& X^{2}=\left(\begin{array}{cc}
256 & -704 \\
0 & 256
\end{array}\right),
\end{aligned}
$$$$
\mathrm{Y}=\left(\begin{array}{cc}
30 & 82 \\
0 & 30
\end{array}\right),
$$$$
Z=\left(\begin{array}{ll}
34 & 62 \\
62 & 34
\end{array}\right)
$$$$
\mathrm{Y}^{2}=\left(\begin{array}{cc}
900 & 4920 \\
0 & 900
\end{array}\right) \text {, }
$$$$
Z^{2}=\left(\begin{array}{cc}
1156 & 4216 \\
0 & 1156
\end{array}\right) \text {, }
$$

And the equality $\left(\mathrm{A}^{\prime \prime}\right)$ is immediately verified.

$\boldsymbol{\beta}$ ) If $\mathrm{a} \neq \mathrm{d}$, then (also) $\mathrm{x} \neq \mathrm{t}$. It follows that:

$$
\mathrm{y}=\frac{\mathrm{b} \cdot(\mathrm{x}-\mathrm{t})}{\mathrm{a}-\mathrm{d}}, \quad \text { with } \quad(\mathrm{a}-\mathrm{d}) \mid \mathrm{b} \cdot(\mathrm{x}-\mathrm{t})
$$

And

$$
A=\left(\begin{array}{ll}
a & b \\
0 & d
\end{array}\right)
$$

and

$$
\mathrm{B}=\left(\begin{array}{cc}
\mathrm{x} & \frac{\mathrm{b} \cdot(\mathrm{x}-\mathrm{t})}{\mathrm{a}-\mathrm{d}} \\
0 & \mathrm{t}
\end{array}\right)
$$

With $a, b, d, x, t \in N, \frac{x-t}{a-d}>0$ and:

$$
\begin{aligned}
& A^{2}=\left(\begin{array}{cc}
a^{2} & b \cdot(a+d) \\
0 & d^{2}
\end{array}\right) \quad \text { and } \quad B^{2}=\left(\begin{array}{cc}
x^{2} & \frac{b \cdot\left(x^{2}-t^{2}\right)}{a-d} \\
0 & t^{2}
\end{array}\right), \\
& A \cdot B=\left(\begin{array}{cc}
a \cdot x & \frac{b \cdot(a \cdot x-d \cdot t)}{a-d} d \\
0 & d \cdot t
\end{array}\right)=B \cdot A .
\end{aligned}
$$


Now, according to the equalities (3.3), we have:

$$
\begin{aligned}
& X=\left(\begin{array}{cc}
a^{2}-x^{2} & \frac{b \cdot\left(a^{2}-d^{2}-x^{2}+t^{2}\right)}{a-d} \\
0 & d^{2}-t^{2}
\end{array}\right), \\
& Y=\left(\begin{array}{cc}
2 \cdot a \cdot x & \frac{2 \cdot b \cdot(a \cdot x-d \cdot t)}{a-d} \\
0 & 2 \cdot d \cdot t
\end{array}\right), \\
& Z=\left(\begin{array}{cc}
a^{2}+x^{2} & \frac{b \cdot\left(a^{2}-d^{2}+x^{2}-t^{2}\right)}{a-d} \\
0 & d^{2}+t^{2}
\end{array}\right) \text {, } \\
& X^{2}=\left(\begin{array}{cc}
\left(a^{2}-x^{2}\right)^{2} & \frac{b \cdot\left(a^{2}-d^{2}-x^{2}+t^{2}\right) \cdot\left(a^{2}+d^{2}-x^{2}-t^{2}\right)}{a-d} \\
0 & \left(d^{2}-t^{2}\right)^{2}
\end{array}\right) \text {, } \\
& \mathrm{Y}^{2}=\left(\begin{array}{cc}
4 \cdot \mathrm{a}^{2} \cdot \mathrm{x}^{2} & \frac{4 \cdot \mathrm{b} \cdot\left(\mathrm{a}^{2} \cdot \mathrm{x}^{2}-\mathrm{d}^{2} \cdot \mathrm{t}^{2}\right)}{\mathrm{a}-\mathrm{d}} \\
0 & 4 \cdot \mathrm{d}^{2} \cdot \mathrm{t}^{2}
\end{array}\right) \text {, } \\
& \mathrm{Z}^{2}=\left(\begin{array}{cc}
\left(\mathrm{a}^{2}+\mathrm{x}^{2}\right)^{2} & \frac{\mathrm{b} \cdot\left(\mathrm{a}^{2}-\mathrm{d}^{2}+\mathrm{x}^{2}-\mathrm{t}^{2}\right) \cdot\left(\mathrm{a}^{2}+\mathrm{d}^{2}+\mathrm{x}^{2}+\mathrm{t}^{2}\right)}{\mathrm{a}-\mathrm{d}} \\
0 & \left(\mathrm{~d}^{2}+\mathrm{t}^{2}\right)^{2}
\end{array}\right),
\end{aligned}
$$

And the equality $\left(\mathrm{A}^{\prime \prime}\right)$ is immediately verified.

Example 3.8.3: Let be the matrices:

$$
\mathrm{A}=\left(\begin{array}{ll}
4 & 5 \\
0 & 3
\end{array}\right) \quad \text { and } \quad \mathrm{B}=\left(\begin{array}{ll}
2 & 5 \\
0 & 1
\end{array}\right) .
$$

Then:

$$
\mathrm{A}^{2}=\left(\begin{array}{cc}
16 & 35 \\
0 & 9
\end{array}\right), \quad \mathrm{B}^{2}=\left(\begin{array}{cc}
4 & 15 \\
0 & 1
\end{array}\right) \quad \text { and } \quad \mathrm{A} \cdot \mathrm{B}=\left(\begin{array}{cc}
8 & 25 \\
0 & 3
\end{array}\right)=\mathrm{B} \cdot \mathrm{A} .
$$

Now, according to the equalities (3.3), we have:

$$
\begin{array}{lll}
X=\left(\begin{array}{cc}
12 & 20 \\
0 & 8
\end{array}\right), & Y=\left(\begin{array}{cc}
16 & 50 \\
0 & 6
\end{array}\right), & Z=\left(\begin{array}{cc}
20 & 50 \\
0 & 10
\end{array}\right), \\
X^{2}=\left(\begin{array}{cc}
144 & 400 \\
0 & 64
\end{array}\right), & Y^{2}=\left(\begin{array}{cc}
256 & 1100 \\
0 & 36
\end{array}\right), & Z^{2}=\left(\begin{array}{cc}
400 & 1500 \\
0 & 100
\end{array}\right),
\end{array}
$$

And the equality $\left(\mathrm{A}^{\prime \prime}\right)$ is immediately verified.

Example 3.8.4: Let be the matrices:

$$
\mathrm{A}=\left(\begin{array}{ll}
5 & 2 \\
0 & 3
\end{array}\right) \quad \text { and } \quad \mathrm{B}=\left(\begin{array}{ll}
9 & 7 \\
0 & 2
\end{array}\right) .
$$

Then:

$$
\mathrm{A}^{2}=\left(\begin{array}{cc}
25 & 16 \\
0 & 9
\end{array}\right), \quad \mathrm{B}^{2}=\left(\begin{array}{cc}
81 & 77 \\
0 & 4
\end{array}\right) \quad \text { and } \quad \mathrm{A} \cdot \mathrm{B}=\left(\begin{array}{cc}
45 & 39 \\
0 & 6
\end{array}\right)=\mathrm{B} \cdot \mathrm{A} \cdot
$$

Now, according to the equalities (3.3), we have:

$$
\begin{array}{lll}
X=\left(\begin{array}{cc}
-56 & -61 \\
0 & 5
\end{array}\right), & Y=\left(\begin{array}{cc}
90 & 78 \\
0 & 12
\end{array}\right), & Z=\left(\begin{array}{cc}
106 & 93 \\
0 & 13
\end{array}\right), \\
X^{2}=\left(\begin{array}{cc}
3136 & 3111 \\
0 & 25
\end{array}\right), & Y^{2}=\left(\begin{array}{cc}
8100 & 7956 \\
0 & 144
\end{array}\right), & Z^{2}=\left(\begin{array}{cc}
11236 & 11067 \\
0 & 169
\end{array}\right),
\end{array}
$$

And the equality $\left(\mathrm{A}^{\prime \prime}\right)$ is immediately verified.

Case 9: $y=z=0, b \neq 0, c \neq 0$. In this case, from the second and third equations of the system (3.6), it follows that:

Then:

$[(\mathrm{b} \cdot \mathrm{x}=\mathrm{b} \cdot \mathrm{t}) \wedge(\mathrm{c} \cdot \mathrm{x}=\mathrm{c} \cdot \mathrm{t})]$

i.e.

$\mathrm{x}=\mathrm{t}$ 


$$
\mathrm{A}=\left(\begin{array}{ll}
\mathrm{a} & \mathrm{b} \\
\mathrm{c} & \mathrm{d}
\end{array}\right) \quad \text { and } \quad \mathrm{B}=\left(\begin{array}{cc}
\mathrm{x} & 0 \\
0 & \mathrm{x}
\end{array}\right)
$$

With a, b, c, d, $\mathbf{x} \in \mathbf{N}$ and:

$$
\begin{array}{ll}
A^{2}=\left(\begin{array}{ll}
a^{2}+b \cdot c & b \cdot(a+d) \\
c \cdot(a+d) & d^{2}+b \cdot c
\end{array}\right) & \text { and } \\
A \cdot B & =\left(\begin{array}{cc}
a \cdot x & 0 \\
c \cdot x & d \cdot x
\end{array}\right)=B \cdot A .
\end{array}
$$

Now, according to the equalities (3.3), we have:

$$
\begin{aligned}
& X=\left(\begin{array}{cc}
a^{2}+b \cdot c-x^{2} & b \cdot(a+d) \\
c \cdot(a+d) & d^{2}+b \cdot c-x^{2}
\end{array}\right), \quad Y=\left(\begin{array}{cc}
2 \cdot a \cdot x & 2 \cdot b \cdot x \\
2 \cdot c \cdot x & 2 \cdot d \cdot x
\end{array}\right), \\
& Z=\left(\begin{array}{cc}
a^{2}+b \cdot c+x^{2} & b \cdot(a+d) \\
c \cdot(a+d) & d^{2}+b \cdot c+x^{2}
\end{array}\right), \\
& X^{2}=\left(\begin{array}{cc}
\left(a^{2}+b \cdot c-x^{2}\right)^{2}+b \cdot c \cdot(a+d)^{2} & b \cdot(a+d) \cdot\left(a^{2}+d^{2}+2 \cdot b \cdot c-2 \cdot x^{2}\right) \\
c \cdot(a+d) \cdot\left(a^{2}+d^{2}+2 \cdot b \cdot c-2 \cdot x^{2}\right) & \left(d^{2}+b \cdot c-x^{2}\right)^{2}+b \cdot c \cdot(a+d)^{2}
\end{array}\right), \\
& Y^{2}=\left(\begin{array}{cc}
4 \cdot a^{2} \cdot x^{2}+4 \cdot b \cdot c \cdot x^{2} & 4 \cdot b \cdot x^{2} \cdot(a+d) \\
4 \cdot c \cdot x^{2} \cdot(a+d) & 4 \cdot d^{2} \cdot x^{2}+4 \cdot b \cdot c \cdot x^{2}
\end{array}\right), \\
& Z^{2}=\left(\begin{array}{cc}
\left(a^{2}+b \cdot c+x^{2}\right)^{2}+b \cdot c \cdot(a+d)^{2} & b \cdot(a+d) \cdot\left(a^{2}+d^{2}+2 \cdot b \cdot c+2 \cdot x^{2}\right) \\
c \cdot(a+d) \cdot\left(a^{2}+d^{2}+2 \cdot b \cdot c+2 \cdot x^{2}\right) & \left(d^{2}+b \cdot c+x^{2}\right)^{2}+b \cdot c \cdot(a+d)^{2}
\end{array}\right),
\end{aligned}
$$

And the equality $\left(\mathrm{A}^{\prime \prime}\right)$ is immediately verified.

Example 3.9.1: Let be the matrices:

$$
\mathrm{A}=\left(\begin{array}{ll}
4 & 1 \\
3 & 5
\end{array}\right) \quad \text { and } \quad \mathrm{B}=\left(\begin{array}{ll}
2 & 0 \\
0 & 2
\end{array}\right) .
$$

Then:

$$
\mathrm{A}^{2}=\left(\begin{array}{cc}
16 & 9 \\
27 & 25
\end{array}\right), \quad \mathrm{B}^{2}=\left(\begin{array}{cc}
4 & 0 \\
0 & 4
\end{array}\right) \quad \text { and } \quad \mathrm{A} \cdot \mathrm{B}=\left(\begin{array}{cc}
8 & 2 \\
6 & 10
\end{array}\right)=\mathrm{B} \cdot \mathrm{A} .
$$

Now, according to the equalities (3.3), we have:

$$
\begin{array}{lll}
X=\left(\begin{array}{cc}
12 & 9 \\
27 & 21
\end{array}\right), & Y=\left(\begin{array}{cc}
16 & 4 \\
12 & 20
\end{array}\right), & Z=\left(\begin{array}{ll}
20 & 9 \\
27 & 29
\end{array}\right), \\
X^{2}=\left(\begin{array}{ll}
144 & 297 \\
891 & 441
\end{array}\right), & Y^{2}=\left(\begin{array}{ll}
256 & 144 \\
432 & 400
\end{array}\right), & Z^{2}=\left(\begin{array}{cc}
400 & 441 \\
1323 & 841
\end{array}\right),
\end{array}
$$

And the equality $\left(\mathrm{A}^{\prime \prime}\right)$ is immediately verified.

Example 3.9.2: Let be the matrices:

$$
\mathrm{A}=\left(\begin{array}{ll}
6 & 1 \\
5 & 7
\end{array}\right) \quad \text { and } \quad \mathrm{B}=\left(\begin{array}{ll}
3 & 0 \\
0 & 3
\end{array}\right) .
$$

Then:

$$
\mathrm{A}^{2}=\left(\begin{array}{ll}
36 & 13 \\
65 & 49
\end{array}\right), \quad \mathrm{B}^{2}=\left(\begin{array}{ll}
9 & 0 \\
0 & 9
\end{array}\right) \quad \text { and } \quad \mathrm{A} \cdot \mathrm{B}=\left(\begin{array}{cc}
18 & 3 \\
15 & 21
\end{array}\right)=\mathrm{B} \cdot \mathrm{A} \text {. }
$$

Now, according to the equalities (3.3), we have:

$$
\begin{array}{lll}
X=\left(\begin{array}{ll}
27 & 13 \\
65 & 40
\end{array}\right), & Y=\left(\begin{array}{cc}
36 & 6 \\
30 & 42
\end{array}\right), & Z=\left(\begin{array}{ll}
45 & 13 \\
65 & 58
\end{array}\right), \\
X^{2}=\left(\begin{array}{cc}
729 & 871 \\
4355 & 1600
\end{array}\right), & Y^{2}=\left(\begin{array}{cc}
1296 & 468 \\
2340 & 1764
\end{array}\right), & Z^{2}=\left(\begin{array}{ll}
2025 & 1339 \\
6695 & 3364
\end{array}\right),
\end{array}
$$


And the equality $\left(\mathrm{A}^{\prime \prime}\right)$ is immediately verified.

Case 10: $y \neq 0, z \neq 0, b \neq 0$ and $c \neq 0$. In this case, of the first (or last) three equations of the system (3.6), it follows that:

$$
\mathrm{z}=\frac{\mathrm{c} \cdot \mathrm{y}}{\mathrm{b}} \text {, with } \mathrm{b} \mid(\mathrm{c} \cdot \mathrm{y}) \quad \text { and } \quad\left\{\begin{array}{l}
\mathrm{y} \cdot(\mathrm{a}-\mathrm{d})=\mathrm{b} \cdot(\mathrm{x}-\mathrm{t}) \\
\mathrm{z} \cdot(\mathrm{a}-\mathrm{d})=\mathrm{c} \cdot(\mathrm{x}-\mathrm{t})
\end{array} .\right.
$$

We distinguish two subcases:

$\alpha$ ) If $\mathrm{a}=\mathrm{d}$, then (also) $\mathrm{x}=\mathrm{t}$. It follows that:

$$
A=\left(\begin{array}{ll}
a & b \\
c & a
\end{array}\right) \quad \text { and } \quad B=\left(\begin{array}{cc}
x & y \\
\frac{c \cdot y}{b} & x
\end{array}\right) \text {, }
$$

With $a, b, c, x, y \in \mathbf{N}$ and:

$$
\begin{aligned}
& A^{2}=\left(\begin{array}{cc}
a^{2}+b \cdot c & 2 \cdot a \cdot b \\
2 \cdot a \cdot c & a^{2}+b \cdot c
\end{array}\right) \quad \text { and } \\
& A \cdot B=\left(\begin{array}{cc}
a \cdot x+c \cdot y & a \cdot y+b \cdot x \\
c \cdot x+\frac{a \cdot c \cdot y}{b} & a \cdot x+c \cdot y
\end{array}\right)=B \cdot A .
\end{aligned}
$$

$$
\mathrm{B}^{2}=\left(\begin{array}{cc}
\mathrm{x}^{2}+\frac{\mathrm{c} \cdot \mathrm{y}^{2}}{\mathrm{~b}} & 2 \cdot \mathrm{x} \cdot \mathrm{y} \\
\frac{2 \cdot \mathrm{x} \cdot \mathrm{y} \cdot \mathrm{c}}{\mathrm{b}} & \mathrm{x}^{2}+\frac{\mathrm{c} \cdot \mathrm{y}^{2}}{\mathrm{~b}}
\end{array}\right)
$$

Now, according to the equalities (3.3), we have:

$$
\begin{aligned}
& X=\left(\begin{array}{cc}
a^{2}+b \cdot c-x^{2}-\frac{c \cdot y^{2}}{b} & 2 \cdot a \cdot b-2 \cdot x \cdot y \\
2 \cdot a \cdot c-\frac{2 \cdot x \cdot y \cdot c}{b} & a^{2}+b \cdot c-x^{2}-\frac{c \cdot y^{2}}{b}
\end{array}\right), \\
& Y=\left(\begin{array}{cc}
2 \cdot a \cdot x+2 \cdot c \cdot y & 2 \cdot a \cdot y+2 \cdot b \cdot x \\
2 \cdot c \cdot x+\frac{2 \cdot a \cdot c \cdot y}{b} & 2 \cdot a \cdot x+2 \cdot c \cdot y
\end{array}\right), \\
& Z=\left(\begin{array}{cc}
a^{2}+b \cdot c+x^{2}+\frac{c \cdot y^{2}}{b} & 2 \cdot a \cdot b+2 \cdot x \cdot y \\
2 \cdot a \cdot c+\frac{2 \cdot x \cdot y \cdot c}{b} & a^{2}+b \cdot c+x^{2}+\frac{c \cdot y^{2}}{b}
\end{array}\right), \\
& X^{2}=\left(\begin{array}{ll}
\alpha & \beta \\
\chi & \alpha
\end{array}\right),
\end{aligned}
$$

Where:

$$
\begin{aligned}
& \alpha=\left(a^{2}+b \cdot c-x^{2}-\frac{c \cdot y^{2}}{b}\right)^{2}+4 \cdot c \cdot(a \cdot b-x \cdot y) \cdot\left(a-\frac{x \cdot y}{b}\right) \\
& \beta=4 \cdot(a \cdot b-x \cdot y) \cdot\left(a^{2}+b \cdot c-x^{2}-\frac{c \cdot y^{2}}{b}\right) \\
& \chi=4 \cdot c \cdot\left(a-\frac{x \cdot y}{b}\right) \cdot\left(a^{2}+b \cdot c-x^{2}-\frac{c \cdot y^{2}}{b}\right) \\
& \delta=4 \cdot(a \cdot x+c \cdot y)^{2}+4 \cdot c \cdot(a \cdot y+b \cdot x) \cdot\left(x+\frac{a \cdot y}{b}\right) \\
& \phi=8 \cdot c \cdot(a \cdot x+c \cdot y) \cdot\left(x+\frac{a \cdot y}{b}\right) \\
& \varphi=\left(a^{2}+b \cdot c+x^{2}+\frac{c \cdot y^{2}}{b}\right)^{2}+4 \cdot c \cdot(a \cdot b+x \cdot y) \cdot\left(a+\frac{x \cdot y}{b}\right)
\end{aligned}
$$




$$
\begin{aligned}
& \gamma=4 \cdot(a \cdot b+x \cdot y) \cdot\left(a^{2}+b \cdot c+x^{2}+\frac{c \cdot y^{2}}{b}\right), \\
& \lambda=4 \cdot c \cdot\left(a+\frac{x \cdot y}{b}\right) \cdot\left(a^{2}+b \cdot c+x^{2}+\frac{c \cdot y^{2}}{b}\right),
\end{aligned}
$$

And the equality $\left(\mathrm{A}^{\prime \prime}\right)$ is immediately verified.

Example 3.10.1: Let be the matrices:

$$
\mathrm{A}=\left(\begin{array}{ll}
4 & 1 \\
3 & 4
\end{array}\right) \quad \text { and } \quad \mathrm{B}=\left(\begin{array}{cc}
2 & 5 \\
15 & 2
\end{array}\right) .
$$

Then:

$$
\mathrm{A}^{2}=\left(\begin{array}{cc}
19 & 8 \\
24 & 19
\end{array}\right), \quad \mathrm{B}^{2}=\left(\begin{array}{cc}
79 & 20 \\
60 & 79
\end{array}\right) \quad \text { and } \quad \mathrm{A} \cdot \mathrm{B}=\left(\begin{array}{ll}
23 & 22 \\
66 & 23
\end{array}\right)=\mathrm{B} \cdot \mathrm{A} \cdot
$$

Now, according to the equalities (3.3), we have:

$$
\begin{array}{lll}
X=\left(\begin{array}{ll}
-60 & -12 \\
-36 & -60
\end{array}\right), & Y=\left(\begin{array}{cc}
46 & 44 \\
132 & 46
\end{array}\right), & Z=\left(\begin{array}{ll}
98 & 28 \\
84 & 98
\end{array}\right), \\
X^{2}=\left(\begin{array}{ll}
4032 & 1440 \\
4320 & 4032
\end{array}\right), & Y^{2}=\left(\begin{array}{cc}
7924 & 4048 \\
12144 & 7924
\end{array}\right), & Z^{2}=\left(\begin{array}{ll}
11956 & 5488 \\
16464 & 11956
\end{array}\right),
\end{array}
$$

And the equality $\left(\mathrm{A}^{\prime \prime}\right)$ is immediately verified.

Example 3.10.2: Let be the matrices:

$$
\mathrm{A}=\left(\begin{array}{ll}
4 & 3 \\
5 & 4
\end{array}\right) \quad \text { and } \quad \mathrm{B}=\left(\begin{array}{cc}
7 & 6 \\
10 & 7
\end{array}\right) .
$$

Then:

$$
\mathrm{A}^{2}=\left(\begin{array}{ll}
31 & 24 \\
40 & 31
\end{array}\right), \quad \mathrm{B}^{2}=\left(\begin{array}{cc}
109 & 84 \\
140 & 109
\end{array}\right) \quad \text { and } \quad \mathrm{A} \cdot \mathrm{B}=\left(\begin{array}{cc}
58 & 45 \\
75 & 58
\end{array}\right)=\mathrm{B} \cdot \mathrm{A} \cdot
$$

Now, according to the equalities (3.3), we have:

$$
\begin{array}{lll}
X=\left(\begin{array}{cc}
-78 & -60 \\
-100 & -78
\end{array}\right), & Y=\left(\begin{array}{cc}
116 & 90 \\
150 & 116
\end{array}\right), & Z=\left(\begin{array}{ll}
140 & 108 \\
180 & 140
\end{array}\right), \\
X^{2}=\left(\begin{array}{cc}
12084 & 9360 \\
15600 & 12084
\end{array}\right), & Y^{2}=\left(\begin{array}{ll}
26956 & 20880 \\
34800 & 26956
\end{array}\right), & Z^{2}=\left(\begin{array}{ll}
39040 & 30240 \\
50400 & 39040
\end{array}\right),
\end{array}
$$

And the equality $\left(\mathrm{A}^{\prime \prime}\right)$ is immediately verified.

$\beta$ ) If $a \neq d$, then (also) $x \neq t$. It follows that:

$$
b \cdot(x-t)=y \cdot(a-d), \quad \text { that is } \quad y=\frac{b \cdot(x-t)}{a-d}, \quad \text { with } \quad(a-d) \mid b \cdot(x-t) .
$$

Hence:

$$
A=\left(\begin{array}{ll}
a & b \\
c & d
\end{array}\right)
$$

and

$$
B=\left(\begin{array}{cc}
x & \frac{b \cdot(x-t)}{a-d} \\
\frac{c \cdot(x-t)}{a-d} & t
\end{array}\right),
$$

With a, b, c, $x, t \in \mathbf{N}, \frac{\mathrm{x}-\mathrm{t}}{\mathrm{a}-\mathrm{d}}>0$ and:

$$
A^{2}=\left(\begin{array}{cc}
a^{2}+b \cdot c & b \cdot(a+d) \\
c \cdot(a+d) & d^{2}+b \cdot c
\end{array}\right) \quad \text { and }
$$




$$
\begin{aligned}
& B^{2}=\left(\begin{array}{cc}
x^{2}+\frac{b \cdot c \cdot(x-t)^{2}}{(a-d)^{2}} & \frac{b \cdot\left(x^{2}-t^{2}\right)}{(a-d)^{2}} \\
\frac{c \cdot\left(x^{2}-t^{2}\right)}{(a-d)^{2}} & t^{2}+\frac{b \cdot c \cdot(x-t)^{2}}{(a-d)^{2}}
\end{array}\right),(3.69) \\
& A \cdot B=\left(\begin{array}{cc}
a \cdot x+\frac{b \cdot c \cdot(x-t)}{a-d} & \frac{b \cdot(a \cdot x-d \cdot t)}{a-d} \\
\frac{c \cdot(a \cdot x-d \cdot t)}{a-d} & d \cdot t+\frac{b \cdot c \cdot(x-t)}{a-d}
\end{array}\right)=B \cdot A .
\end{aligned}
$$

Now, according to the equalities (3.3), we have:

$$
\begin{aligned}
& X=\left(\begin{array}{cc}
a^{2}+b \cdot c-x^{2}-\frac{b \cdot c \cdot\left(x^{2}-t^{2}\right)}{(a-d)^{2}} & b \cdot(a+d)-\frac{b \cdot\left(x^{2}-t^{2}\right)}{a-d} \\
c \cdot(a+d)-\frac{c \cdot\left(x^{2}-t^{2}\right)}{a-d} & d^{2}+b \cdot c-t^{2}-\frac{b \cdot c \cdot\left(x^{2}-t^{2}\right)}{(a-d)^{2}}
\end{array}\right), \\
& Y=\left(\begin{array}{cc}
2 \cdot a \cdot x+\frac{2 \cdot b \cdot c \cdot(x-t)}{a-d} & \frac{2 \cdot b \cdot(a \cdot x-d \cdot t)}{a-d} \\
\frac{2 \cdot c \cdot(a \cdot x-d \cdot t)}{a-d} & 2 \cdot d \cdot t+\frac{2 \cdot b \cdot c \cdot(x-t)}{a-d}
\end{array}\right), \\
& Z=\left(\begin{array}{cc}
a^{2}+b \cdot c+x^{2}+\frac{b \cdot c \cdot\left(x^{2}-t^{2}\right)}{(a-d)^{2}} & b \cdot(a+d)+\frac{b \cdot\left(x^{2}-t^{2}\right)}{a-d} \\
c \cdot(a+d)+\frac{c \cdot\left(x^{2}-t^{2}\right)}{a-d} & d^{2}+b \cdot c+t^{2}+\frac{b \cdot c \cdot\left(x^{2}-t^{2}\right)}{(a-d)^{2}}
\end{array}\right), \\
& X^{2}=\left(\begin{array}{ll}
\alpha & \beta \\
\chi & \delta
\end{array}\right), \quad Y^{2}=\left(\begin{array}{ll}
\varepsilon & \phi \\
\varphi & \gamma
\end{array}\right),
\end{aligned}
$$

Where:

$$
\begin{aligned}
& \alpha=\left(a^{2}+b \cdot c-x^{2}-\frac{b \cdot c \cdot(x-t)^{2}}{(a-d)^{2}}\right)^{2}+b \cdot c \cdot\left(a+d-\frac{x^{2}-t^{2}}{a-d}\right)^{2} \\
& \beta=b \cdot\left(a^{2}+d^{2}+2 \cdot b \cdot c-x^{2}-t^{2}-\frac{2 \cdot b \cdot c \cdot\left(x^{2}-t^{2}\right)}{(a-d)^{2}}\right)\left(a+d-\frac{x^{2}-t^{2}}{a-d}\right) \\
& \chi=c \cdot\left(a^{2}+d^{2}+2 \cdot b \cdot c-x^{2}-t^{2}-\frac{2 \cdot b \cdot c \cdot\left(x^{2}-t^{2}\right)}{(a-d)^{2}}\right)\left(a+d-\frac{x^{2}-t^{2}}{a-d}\right) \\
& \delta=\left(d^{2}+b \cdot c-t^{2}-\frac{b \cdot c \cdot(x-t)^{2}}{(a-d)^{2}}\right)^{2}+b \cdot c \cdot\left(a+d-\frac{x^{2}-t^{2}}{a-d}\right)^{2} \\
& \varepsilon=4 \cdot\left(a \cdot x+\frac{b \cdot c \cdot(x-t)}{a-d}\right)^{2}+4 \cdot \frac{b \cdot c \cdot(a \cdot x-d \cdot t)^{2}}{(a-d)^{2}}, \\
& \phi=4 \cdot b \cdot\left(a \cdot x+d \cdot t+\frac{2 \cdot b \cdot c \cdot(x-t)}{a-d}\right)^{2} \cdot \frac{(a \cdot x-d \cdot t)}{a-d} \\
& \varphi=4 \cdot c \cdot\left(a \cdot x+d \cdot t+\frac{2 \cdot b \cdot c \cdot(x-t)}{a-d}\right)^{2} \cdot \frac{(a \cdot x-d \cdot t)}{a-d} \\
& \gamma=4 \cdot\left(d \cdot t+\frac{b \cdot c \cdot(x-t)}{a-d}\right)^{2}+4 \cdot \frac{b \cdot c \cdot(a \cdot x-d \cdot t)^{2}}{(a-d)^{2}}, \\
& \eta=\left(a{ }^{2}+b \cdot c+x+\frac{b \cdot c \cdot(x-t)^{2}}{(a-d)}\right)^{2}+b \cdot c \cdot\left(a+d+\frac{x^{2}-t^{2}}{a-d}\right)^{2}
\end{aligned}
$$




$$
\begin{aligned}
& \mathrm{l}=\mathrm{b} \cdot\left(\mathrm{a}^{2}+\mathrm{d}^{2}+2 \cdot \mathrm{b} \cdot \mathrm{c}+\mathrm{x}^{2}+\mathrm{t}^{2}+\frac{2 \cdot \mathrm{b} \cdot \mathrm{c} \cdot\left(\mathrm{x}^{2}-\mathrm{t}^{2}\right)}{(\mathrm{a}-\mathrm{d})^{2}}\right)\left(\mathrm{a}+\mathrm{d}+\frac{\mathrm{x}^{2}-\mathrm{t}^{2}}{\mathrm{a}-\mathrm{d}}\right), \\
& \kappa=\mathrm{c} \cdot\left(\mathrm{a}^{2}+\mathrm{d}^{2}+2 \cdot \mathrm{b} \cdot \mathrm{c}+\mathrm{x}^{2}+\mathrm{t}^{2}+\frac{2 \cdot \mathrm{b} \cdot \mathrm{c} \cdot\left(\mathrm{x}^{2}-\mathrm{t}^{2}\right)}{(\mathrm{a}-\mathrm{d})^{2}}\right)\left(\mathrm{a}+\mathrm{d}+\frac{\mathrm{x}^{2}-\mathrm{t}^{2}}{\mathrm{a}-\mathrm{d}}\right), \\
& \lambda=\left(\mathrm{d}^{2}+\mathrm{b} \cdot \mathrm{c}+\mathrm{t}^{2}+\frac{\mathrm{b} \cdot \mathrm{c} \cdot(\mathrm{x}-\mathrm{t})^{2}}{(\mathrm{a}-\mathrm{d})^{2}}\right)^{2}+\mathrm{b} \cdot \mathrm{c} \cdot\left(\mathrm{a}+\mathrm{d}+\frac{\mathrm{x}^{2}-\mathrm{t}^{2}}{\mathrm{a}-\mathrm{d}}\right)^{2} .
\end{aligned}
$$

And the equality $\left(\mathrm{A}^{\prime \prime}\right)$ is immediately verified.

Example 3.10.3: Let be the matrices:

$$
\mathrm{A}=\left(\begin{array}{ll}
4 & 5 \\
2 & 3
\end{array}\right) \quad \text { and } \mathrm{B}=\left(\begin{array}{cc}
5 & 10 \\
4 & 3
\end{array}\right) .
$$

Then:

$$
\mathrm{A}^{2}=\left(\begin{array}{ll}
26 & 35 \\
14 & 19
\end{array}\right), \quad \mathrm{B}^{2}=\left(\begin{array}{cc}
65 & 80 \\
32 & 49
\end{array}\right) \quad \text { and } \quad \mathrm{A} \cdot \mathrm{B}=\left(\begin{array}{ll}
40 & 55 \\
22 & 29
\end{array}\right)=\mathrm{B} \cdot \mathrm{A}
$$

Now, according to the equalities (3.3), we have:

$$
\begin{array}{lll}
X=\left(\begin{array}{ll}
-39 & -45 \\
-18 & -30
\end{array}\right), & Y=\left(\begin{array}{cc}
80 & 110 \\
44 & 58
\end{array}\right), & Z=\left(\begin{array}{cc}
91 & 115 \\
46 & 68
\end{array}\right), \\
X^{2}=\left(\begin{array}{ll}
2331 & 3105 \\
1242 & 1710
\end{array}\right), & Y^{2}=\left(\begin{array}{cc}
11240 & 15180 \\
6072 & 8204
\end{array}\right), & Z^{2}=\left(\begin{array}{cc}
13571 & 18285 \\
7314 & 9914
\end{array}\right),
\end{array}
$$

And the equality $\left(\mathrm{A}^{\prime \prime}\right)$ is immediately verified.

Example 3.10.4: Let be the matrices:

$$
\mathrm{A}=\left(\begin{array}{ll}
5 & 3 \\
7 & 2
\end{array}\right) \quad \text { and } \quad \mathrm{B}=\left(\begin{array}{cc}
9 & 6 \\
14 & 3
\end{array}\right) .
$$

Then:

$$
\mathrm{A}^{2}=\left(\begin{array}{ll}
46 & 21 \\
49 & 25
\end{array}\right), \quad \mathrm{B}^{2}=\left(\begin{array}{cc}
165 & 72 \\
168 & 93
\end{array}\right) \quad \text { and } \quad \mathrm{A} \cdot \mathrm{B}=\left(\begin{array}{ll}
87 & 39 \\
91 & 48
\end{array}\right)=\mathrm{B} \cdot \mathrm{A}
$$

Now, according to the equalities (3.3), we have:

$$
\begin{array}{lll}
X=\left(\begin{array}{ll}
-119 & -51 \\
-119 & -68
\end{array}\right), & Y=\left(\begin{array}{ll}
174 & 78 \\
182 & 96
\end{array}\right), & Z=\left(\begin{array}{cc}
211 & 93 \\
217 & 118
\end{array}\right), \\
X^{2}=\left(\begin{array}{ll}
20230 & 9537 \\
22253 & 10693
\end{array}\right), & Y^{2}=\left(\begin{array}{ll}
44472 & 21060 \\
49140 & 23412
\end{array}\right), & Z^{2}=\left(\begin{array}{ll}
64702 & 30597 \\
71393 & 34105
\end{array}\right),
\end{array}
$$

And the equality $\left(\mathrm{A}^{\prime \prime}\right)$ is immediately verified.

Now we can say that we have completely solved the system (3.6) and, implicitly, we determined all matrix pairs $(\mathrm{A}, \mathrm{B}) \in \mathscr{M}_{2}(\mathbf{N}) \times \mathscr{M}_{2}(\mathbf{N})$, which satisfy the equalities (3.2) and (3.1) and which, according to the equalities (3.3), lead to solutions $(\mathrm{X}, \mathrm{Y}, \mathrm{Z}) \in \mathscr{\mathscr { M }}_{2}(\mathbf{Z}) \times \mathscr{\mathscr { M }}_{2}(\mathbf{Z}) \times \mathscr{\mathscr { M }}_{2}(\mathbf{Z})$ of the equation $\left(\mathrm{A}^{\prime \prime}\right)$.

\section{Conclusions}

There it is, therefore, that any equation of form (A) can be translated into the ring $\left(\mathscr{M}_{2}(\mathbf{Z}),+, \cdot\right)$ - becoming the equation $\left(\mathrm{A}^{\prime \prime}\right)$, which has as solutions matrices of the form (3.3), and matrices A and B first verify the equalities (3.2) and (3.1'). According to those presented in the previous paragraph, moreover these matrices A and B need to verify exactly one of the equalities (3.8), (3.13), (3.18), (3.23), (3.28), (3.33), (3.38), (3.43), (3.48), (3.53), (3.58), (3.63), (3.68).

As mentioned above, pairs of matrices $(\mathrm{A}, \mathrm{B})$ which satisfy one of these 13 equalities are the only ones which satisfy and the equalities (3.2) and (3.1'). But, naturally, the question arises:

„Apart from the solutions given by equality (3.3), there are others matrix triplets $(X, Y, Z) \in \mathscr{M T}_{2}(\mathbf{Z}) \times \mathscr{M T}_{2}(\mathbf{Z}) \times \mathscr{M}_{2}(Z)$, of another form, which are solutions of the equation ( $A$ ")?" 
Let's try to answer this question. We consider the matrices $\mathrm{X}, \mathrm{Y}, \mathrm{Z} \in \mathscr{\mathscr { M }}_{2}(\mathbf{Z})$, where:

$$
X=\left(\begin{array}{ll}
a & b \\
c & d
\end{array}\right), \quad Y=\left(\begin{array}{cc}
m & n \\
p & q
\end{array}\right) \quad \text { and } \quad Z=\left(\begin{array}{ll}
\alpha & \beta \\
\gamma & \delta
\end{array}\right) \text {, }
$$

with a, b, c, d, m, n, p, q, $\alpha, \beta, \gamma, \delta \in \mathbf{N}$.

The equality $\left(\mathrm{A}^{\prime \prime}\right)$ implies the system:

$$
\left\{\begin{array}{l}
a^{2}+b \cdot c+m^{2}+n \cdot p=\alpha^{2}+\beta \cdot \lambda \\
a \cdot b+b \cdot d+n \cdot m+n \cdot q=\alpha \cdot \beta+\beta \cdot \delta \\
a \cdot c+c \cdot d+p \cdot m+p \cdot q=\alpha \cdot \gamma+\lambda \cdot \delta \\
d^{2}+b \cdot c+q^{2}+n \cdot p=\delta^{2}+\beta \cdot \lambda
\end{array} .\right.
$$

From the first and last equation of the system (4.2) it follows that:

$$
a^{2}+m^{2}+\delta^{2}=d^{2}+q^{2}+\alpha^{2} \text {. }
$$

Considering the equalities:

$$
\mathrm{a}^{2}+\mathrm{m}^{2}=\alpha^{2} \quad \text { and } \quad \mathrm{d}^{2}+\mathrm{q}^{2}=\delta^{2},\left(4.3^{\prime}\right)
$$

we obtain the following solution of the equation (4.3):

$$
(\mathrm{a}, \mathrm{d}, \mathrm{m}, \mathrm{q}, \alpha, \delta)=(3,6,4,8,5,10) \text {. }
$$

From the second and third equations of the system (4.2) follows equalities:

$$
\left\{\begin{array}{l}
\mathrm{b} \cdot(\mathrm{a}+\mathrm{d})+\mathrm{n} \cdot(\mathrm{m}+\mathrm{q})=\beta \cdot(\alpha+\delta) \\
\mathrm{c} \cdot(\mathrm{a}+\mathrm{d})+\mathrm{p} \cdot(\mathrm{m}+\mathrm{q})=\gamma \cdot(\alpha+\delta)
\end{array},\right.
$$

which, under the conditions of equality (4.4), become:

$$
\left\{\begin{array}{l}
3 \cdot b+4 \cdot n=5 \cdot \beta \\
3 \cdot c+4 \cdot p=5 \cdot \gamma
\end{array}\right.
$$

A solution of the system (4.5') is:

$$
(\mathrm{b}, \mathrm{c}, \mathrm{n}, \mathrm{p}, \beta, \gamma)=(5,3,5,4,7,5) \text {. }
$$

From equalities (4.4) and (4.6), the equalities (4.1) become:

$$
\mathrm{X}=\left(\begin{array}{ll}
3 & 5 \\
3 & 6
\end{array}\right), \quad \mathrm{Y}=\left(\begin{array}{ll}
4 & 5 \\
4 & 8
\end{array}\right) \quad \text { and } \quad \mathrm{Z}=\left(\begin{array}{cc}
5 & 7 \\
5 & 10
\end{array}\right) .
$$

Then:

$$
\mathrm{X}^{2}=\left(\begin{array}{ll}
24 & 45 \\
27 & 51
\end{array}\right), \quad \mathrm{Y}^{2}=\left(\begin{array}{ll}
36 & 60 \\
48 & 84
\end{array}\right) \quad \text { and } \quad Z^{2}=\left(\begin{array}{ll}
60 & 105 \\
75 & 135
\end{array}\right) .
$$

We remark that matrices from equalities (4.7) satisfy equality $\left(\mathrm{A}^{\prime \prime}\right)$. Now we assume that there are two matrices A, $\mathrm{B} \in \mathscr{M}_{2}(\mathbf{N})$, which satisfy the equalities (3.2), respectively (3.3). Then:

$$
\mathrm{A}^{2}=\left(\begin{array}{ll}
4 & 6 \\
4 & 8
\end{array}\right)
$$

Where from obtain the system:

$$
\left\{\begin{array}{l}
a^{2}+b \cdot c=4 \\
b \cdot(a+d)=6 \\
c \cdot(a+d)=4 \\
d^{2}+b \cdot c=8
\end{array} .\right.
$$

From the first and last equation of the system (4.9) it follows that:

$$
\mathrm{a}=0 \quad \text { and } \mathrm{d}=2 \text {, }
$$

and then, from the second and third equations, it follows that:

$$
\mathrm{b}=3 \text { and } \mathrm{c}=2 \text {. }
$$

It is noted that this solution does not satisfy neither the first nor the last equation of the system (4.9). Therefore, there are not two matrices $\mathrm{A}, \mathrm{B} \in \mathscr{\mathscr { M }}_{2}(\mathbf{N})$, which satisfy the equalities (3.2), respectively (3.3); or, in other words, the solution $(\mathrm{X}, \mathrm{Y}, \mathrm{Z}) \in \mathscr{M}_{2}(\mathbf{Z}) \times \mathscr{M}_{2}(\mathbf{Z}) \times \mathscr{M}_{2}(\mathbf{Z})$, from (4.1') is not the form (3.3). In conclusion, the answer to question $(\Lambda)$ is YES. 
We can say that if to equation (A) we determined, in Paragraph 2, all the solutions to equation (A") we have not determined them all.

On the other hand, it can be seen that we can consider the matrices A and B in the ring $\left(\mathscr{M}_{\mathrm{n}}(\mathbf{Z}),+, \cdot\right)$, with $\mathrm{n} \in \mathbf{N}^{*}$, any number, at least equal to 2 . We have considered the ring $\left(\mathscr{M}_{2}(\mathbf{Z}),+, \cdot\right)$ only because the equality $\left(3.1^{\prime}\right)$ leads to a more resolvable system in the set of natural numbers. Of course, for any natural number $n$, at least equal to 2 , the system (3.6) has exactly $n^{2}$ equations and all unknown, and if $n \geq 3$, then solving it, even in the set $\mathbf{N}$ of natural numbers, is quite difficult.

Without insisting on the calculations, present further an example solution of the equation $\left(\mathrm{A}^{\prime \prime}\right)$ from the set $\mathscr{\mathscr { M }}_{3}(\mathbf{Z})$.

Example 4.1: Let be the matrices:

$$
\mathrm{A}=\left(\begin{array}{ccc}
-1 & 2 & 4 \\
-6 & 5 & 2 \\
10 & 8 & 7
\end{array}\right) \quad \text { and } \mathrm{B}=\left(\begin{array}{ccc}
-1 & 1 & 2 \\
-3 & 2 & 1 \\
5 & 4 & 3
\end{array}\right)
$$

Then:

$$
\mathrm{A}^{2}=\left(\begin{array}{ccc}
29 & 40 & 28 \\
-4 & 29 & 0 \\
12 & 116 & 105
\end{array}\right), \quad \mathrm{B}^{2}=\left(\begin{array}{ccc}
8 & 9 & 5 \\
2 & 5 & -1 \\
-2 & 25 & 23
\end{array}\right) \quad \text { and } \quad \mathrm{A} \cdot \mathrm{B}=\left(\begin{array}{ccc}
15 & 19 & 12 \\
1 & 12 & -1 \\
1 & 54 & 49
\end{array}\right)=\mathrm{B} \cdot \mathrm{A}
$$

Now, according to the equalities (3.3), we have:

$$
\begin{aligned}
& \mathrm{X}=\left(\begin{array}{ccc}
21 & 31 & 23 \\
-6 & 24 & 1 \\
14 & 91 & 82
\end{array}\right), \quad \mathrm{Y}=\left(\begin{array}{ccc}
30 & 38 & 24 \\
2 & 24 & -2 \\
2 & 108 & 98
\end{array}\right), \quad \mathrm{Z}=\left(\begin{array}{ccc}
37 & 49 & 33 \\
-2 & 34 & -1 \\
10 & 141 & 128
\end{array}\right) \text {, } \\
& X^{2}=\left(\begin{array}{ccc}
577 & 3488 & 2400 \\
-256 & 481 & -32 \\
896 & 10080 & 7137
\end{array}\right), \quad Y^{2}=\left(\begin{array}{ccc}
1024 & 4644 & 2996 \\
104 & 436 & -196 \\
472 & 13252 & 9436
\end{array}\right), \quad Z^{2}=\left(\begin{array}{ccc}
1601 & 8132 & 5396 \\
-152 & 917 & -228 \\
1368 & 23332 & 16573
\end{array}\right),
\end{aligned}
$$

And the equality $\left(\mathrm{A}^{\prime \prime}\right)$ is immediately verified.

However, we can say that every solution of those determined in Paragraph 2 induces a solution $\left(\mathrm{X}^{(\mathrm{n})}, \mathrm{Y}^{(\mathrm{n})}, \mathrm{Z}^{(\mathrm{n})}\right) \in \mathscr{M}_{\mathrm{n}}(\mathbf{Z}) \times \mathscr{M}_{\mathrm{n}}(\mathbf{Z}) \times \mathscr{M}_{\mathrm{n}}(\mathbf{Z})$. Indeed, if A, B $\in \mathscr{M}_{2}(\mathbf{N})$ satisfy the equalities (2.2) and (2.3), then:

$$
A^{(n)}=\left(\begin{array}{cc}
A & O_{2, n-2} \\
O_{n-2,2} & O_{n-2, n-2}
\end{array}\right) \quad \text { and } \quad B^{(n)}=\left(\begin{array}{cc}
B & O_{2, n-2} \\
O_{n-2,2} & O_{n-2, n-2}
\end{array}\right)
$$

Are two matrices of $\mathscr{M}_{\mathrm{n}}(\mathbf{N})$, which satisfy the equalities (3.3). Now, it is easily verified that matrices:

$$
\mathrm{X}^{(\mathrm{n})}=\left(\mathrm{A}^{(\mathrm{n})}\right)^{2}-\left(\mathrm{B}^{(\mathrm{n})}\right)^{2}, \quad \mathrm{Y}^{(\mathrm{n})}=2 \cdot \mathrm{A}^{(\mathrm{n})} \cdot \mathrm{B}^{(\mathrm{n})} \text { and } \quad \mathrm{Z}^{(\mathrm{n})}=\left(\mathrm{A}^{(\mathrm{n})}\right)^{2}+\left(\mathrm{B}^{(\mathrm{n})}\right)^{2},
$$

From $\mathscr{M}_{\mathrm{n}}(\mathbf{Z})$ are solutions of equation $\left(\mathrm{A}^{\prime \prime}\right)$.

Example 4.2: Let us consider matrices:

$$
\mathrm{A}=\left(\begin{array}{ll}
5 & 3 \\
7 & 2
\end{array}\right) \quad \text { and } \mathrm{B}=\left(\begin{array}{cc}
9 & 6 \\
14 & 3
\end{array}\right) \text {, }
$$

of Example 3.10.4. Then, for:

$\mathrm{n}=5$,

According to the equalities (4.12), these matrices induce matrices:

$$
\mathrm{A}^{(5)}=\left(\begin{array}{cc}
\mathrm{A} & \mathrm{O}_{2,3} \\
\mathrm{O}_{3,2} & \mathrm{O}_{3,3}
\end{array}\right)=\left(\begin{array}{ccccc}
5 & 3 & 0 & 0 & 0 \\
7 & 2 & 0 & 0 & 0 \\
0 & 0 & 0 & 0 & 0 \\
0 & 0 & 0 & 0 & 0 \\
0 & 0 & 0 & 0 & 0
\end{array}\right) \quad \text { and } \quad \mathrm{B}^{(5)}=\left(\begin{array}{cc}
\mathrm{B} & \mathrm{O}_{2,3} \\
\mathrm{O}_{3,2} & \mathrm{O}_{3,3}
\end{array}\right)=\left(\begin{array}{ccccc}
9 & 6 & 0 & 0 & 0 \\
14 & 3 & 0 & 0 & 0 \\
0 & 0 & 0 & 0 & 0 \\
0 & 0 & 0 & 0 & 0 \\
0 & 0 & 0 & 0 & 0
\end{array}\right) \text {. }
$$

Then: 


$$
\left(A^{(5)}\right)^{2}=\left(\begin{array}{ccccc}
46 & 21 & 0 & 0 & 0 \\
49 & 25 & 0 & 0 & 0 \\
0 & 0 & 0 & 0 & 0 \\
0 & 0 & 0 & 0 & 0 \\
0 & 0 & 0 & 0 & 0
\end{array}\right)
$$

and

Now, according to equalities (3.3') we have:

$$
\begin{aligned}
& \mathrm{X}^{(5)}=\left(\begin{array}{ccccc}
-119 & -51 & 0 & 0 & 0 \\
-119 & -68 & 0 & 0 & 0 \\
0 & 0 & 0 & 0 & 0 \\
0 & 0 & 0 & 0 & 0 \\
0 & 0 & 0 & 0 & 0
\end{array}\right), \mathrm{Y}^{(5)}=\left(\begin{array}{cccccc}
174 & 78 & 0 & 0 & 0 \\
182 & 96 & 0 & 0 & 0 \\
0 & 0 & 0 & 0 & 0 \\
0 & 0 & 0 & 0 & 0 \\
0 & 0 & 0 & 0 & 0
\end{array}\right), \quad \mathrm{Z}^{(5)}=\left(\begin{array}{ccccc}
11 & 93 & 0 & 0 & 0 \\
217 & 118 & 0 & 0 & 0 \\
0 & 0 & 0 & 0 & 0 \\
0 & 0 & 0 & 0 & 0 \\
0 & 0 & 0 & 0 & 0
\end{array}\right), \\
& \left(\mathrm{X}^{(5)}\right)^{2}=\left(\begin{array}{ccccc}
20230 & 9537 & 0 & 0 & 0 \\
22253 & 10693 & 0 & 0 & 0 \\
0 & 0 & 0 & 0 & 0 \\
0 & 0 & 0 & 0 & 0 \\
0 & 0 & 0 & 0 & 0
\end{array}\right), \\
& \left(\mathrm{Z}^{(\mathrm{n})}\right)^{2}=\left(\begin{array}{cccccc}
64702 & 30597 & 0 & 0 & 0 \\
71393 & 34105 & 0 & 0 & 0 \\
0 & 0 & 0 & 0 & 0 \\
0 & 0 & 0 & 0 & 0 \\
0 & 0 & 0 & 0 & 0
\end{array}\right),
\end{aligned}
$$$$
\mathrm{A}^{(5)} \cdot \mathrm{B}^{(5)}=\left(\begin{array}{ccccc}
87 & 39 & 0 & 0 & 0 \\
91 & 48 & 0 & 0 & 0 \\
0 & 0 & 0 & 0 & 0 \\
0 & 0 & 0 & 0 & 0 \\
0 & 0 & 0 & 0 & 0
\end{array}\right)=\mathrm{B}^{(5)} \cdot \mathrm{A}^{(5)} \text {. }
$$

and the equality $\left(\mathrm{A}^{\prime \prime}\right)$ is immediately verified.

Of course we can reformulate Corollary 2.9 and matrix solutions as well:

Corollary 4.3: If $X, Y, Z \in M_{n}(Z)$, is a solution of the equation ( $\left.A^{\prime \prime}\right)$ and:

then:

$$
X \cdot Y=Y \cdot X, \quad Y \cdot Z=Z \cdot Y \quad \text { and } \quad X \cdot Z=Z X,(4.13)
$$

$$
T=X+2 \cdot Y-2 \cdot Z, \quad U=2 \cdot X+Y-2 \cdot Z, \quad \text { and } \quad Z=2 \cdot X+2 \cdot Y-3 \cdot Z, \quad(* * *)
$$

is also the solution of the same equations.

Definition 4.4:Any three matrices $X, Y, Z \in M_{n}(Z)$, which represent a solution of the equation (A") will be called Pythagorean (or pitagoric) matrices.

As a general conclusion, we can say that any equation of form (A) can "sink" into a ring of matrices of the type $\left(\mathscr{\mathscr { M }}_{\mathrm{n}}(\mathbf{Z}),+, \cdot\right)$, with $\mathrm{n} \in \mathbf{N}^{*}$, an arbitrary number, at least equal to 2 ; only that, if equation (A) can be solved completely in the ring of integers $(\mathbf{Z},+, \cdot)$, that is, all its solutions can be determined, the same can not be said about the "submerged" equation $\left(\mathrm{A}^{\prime \prime}\right)$, that is, in this matrix ring the determination of all solutions is usually quite difficult and then only certain solutions are determined, such as those in Paragraph 2.

Of course this paper is one of Didactics of Mathematics and addresses both students, students or teachers who are interested and interested in these issues, to whom we believe we have formed, in this way, a good image of solving these two types of equations.

The notations we used were those indatabasehttp://mathworld.wolfram.com/topics/Algebra.html. 


\section{References}

Acu, D.,Aritmeticasiteorianumerelor (Arithmetic and Number Theory), EdituraUniversitatii „Lucian Blaga”, Sibiu, 2010.

Andreescu, T., Andrica, D.,O introducere in studiulecuațiilordiofantiene (An Introduction to the Study of Diofantian Equations), Editura GIL, Zalău, 2002.

Borevici, Z. I., Safarevici, I. R.,TeoriaNumerelor (Number theory), EdituraStiințificășiEnciclopedică, București, 1985.

Cosniță, C., Turtoiu, F.,Problemede algebră (Algebraproblems), EdituraTehnică, Bucuresti, 1989.

Cucurezeanu, I.,Problemede aritmeticăşiteorianumerelor (Arithmeticproblems and numbertheory), EdituraTehnică, București, 1976.

Cucurezeanu, I.,Ecuaţiiînnumereîntregi (Equations in integernumbers), EdituraAramisPrint, Bucureşti, 2006.

Ion D. Ion, Niță, C., Năstăsescu, C.,Complementede algebră (Complements of algebra), EdituraStiințificășiEnciclopedică, București, 1984.

Ion. D. Ion., Niță, C., Radu, N., Popescu, D.,Problemede algebră (Algebraproblems), EdituraDidacticășiPedagogică, București, 1981.

Năstăsecu, C., Andrei, G., Țena, M., Otărășanu, I.,Probleme de structurialgebrice (Problems of algebraic structures), EdituraAcademieiRepublicii Socialiste România, Bucuresti, 1988.

Vălcan, D.,FORMAREA INIŢIALA A PROFESORILOR DE MATEMATICĂ LA UNIVERSITATEA „BABEŞ-BOLYAI”, NIVEL LICENŢĂ, CurriculumulNaționalmatematicpreuniversitarînperioada 2000 - 2016, Vol. I: De la obiective / competențe la planuri - cadru de învățământ (INITIAL TRAINING OF MATHEMATICS TEACHERS IN "BABESS-BOLYAI" UNIVERSITY, LICENSE LEVEL, National Pre - University Mathematical Curriculum in the Period 2000 - 2016, Vol. I: From Objectives / Competencies to curriculum framework plans), Editura Casa Cărţii de Ştiinţă, Cluj-Napoca, 2017.

http://mathworld.wolfram.com/topics/Algebra.html 